\title{
Acid Mine Drainage Remediation: Aluminum Chelation Using Functional Graphenic Materials
}

Michelle M. Karpinsky, ${ }^{\mathrm{a} 广}$ Anne M. Arnold, ${ }^{\mathrm{b}}{ }^{\dagger}$ Jaejun Lee, ${ }^{\mathrm{c}}$ Genell Jasper, ${ }^{\mathrm{a}}$ Michael R. Bockstaller, ${ }^{\mathrm{c}}$ Stefanie A. Sydlik, ${ }^{\text {d,e }}$ Edward P. Zovinka ${ }^{a}$

${ }^{a}$ Department of Chemistry, Saint Francis University, Loretto, PA 15940, USA

${ }^{b}$ Chemical and Biological Signature Sciences Group, National Security Directorate, Pacific

Northwest National Laboratory, Richland, WA 99354, USA

${ }^{c}$ Department of Materials Science and Engineering, Carnegie Mellon University, Pittsburgh, PA 15213, USA

${ }^{d}$ Department of Chemistry, Carnegie Mellon University, Pittsburgh, PA 15213, USA

${ }^{e}$ Department of Biomedical Engineering, Carnegie Mellon University, Pittsburgh, PA 15213, USA

† These authors contributed equally

\section{Supporting Information}

\author{
Corresponding Authors \\ ezovinka@francis.edu \\ ssydlik@andrew.cmu.edu
}




\section{Table of Contents}

1. Supplemental Materials and Methods

$(\mathrm{S} 4-\mathrm{S} 10)$

1.1 Synthetic Methods

$(\mathrm{S} 4-\mathrm{S} 6)$

1.2 Material Characterization

$(\mathrm{S} 6-\mathrm{S} 8)$

1.3 FGM Aluminum Chelation

(S8)

1.4 FGM Regeneration

1.5 FGM Stability in Acidic Water

(S8)

1.6 FGM Bacterial Culture

$(\mathrm{S} 8-\mathrm{S} 10)$

\section{Supplemental Results and Discussion}

2.1 Hydroxamic Acid Functionalized FGMs

$(\mathrm{S} 11-\mathrm{S} 12)$

2.2 Catechol Functionalized FGMs

(S11)

2.3 Chelation Capacity and Regeneration of FGMs

$(\mathrm{S} 11-\mathrm{S} 12)$

2.4 Ecological Impact of FGMs

3. Supplemental Reaction Schemes

$(\mathrm{S} 13-\mathrm{S} 14)$

Scheme S1. GO Synthesis

Scheme S2. CG, HA-GO, HA-CG, CGO, and CCG Synthesis

\section{Supplemental Figures}

$(\mathrm{S} 15-\mathrm{S} 44)$

Figure S1. FTIR Spectroscopy of As-Synthesized FGMs

Figure S2. Elemental XPS of All FGMs

Figure S3. High Resolution XPS C1s of GO and CG

Figure S5. ${ }^{1} \mathrm{H}-\mathrm{NMR}$ Spectroscopy of GO, CG, HA-GO, and

$$
\text { HA-CG Washes }
$$

Figure S6. High Resolution XPS N1s of CGO and CCG

Figure S8. Elemental XPS of All FGMs After Aluminum

Exposure

Figure S9. Edge- and Basal Plane-Aluminum Chelation

Schematic

Figure S10. Elemental and High Resolution A12p XPS of

All FGMs After Aluminum Regeneration

Figure S11. Elemental XPS of All FGMs After 1 Week

In AMD-like Conditions

Figure S12. High Resolution XPS C1s of GO and CG After

1 Week In AMD-like Conditions

Figure S13. High Resolution XPS N1s of CGO and CCG

After 1 Week In AMD-like Conditions

Figure S14. LIVE/DEAD ${ }^{\circledR}$ BacLight ${ }^{\mathrm{TM}}$ Standard Curve

Figure S15. Absorption Spectroscopy of FGM Control

Figure S16. Fluorescence Spectroscopy of LIVE/DEAD ${ }^{\circledR}$

Figure S17. LIVE/DEAD ${ }^{\circledR}$ BacLight ${ }^{\mathrm{TM}}$ Fluorescence Images

of GO-Treated $E$. coli

(S33 - S34) 
Figure S18. LIVE/DEAD ${ }^{\circledR}$ BacLight ${ }^{\mathrm{TM}}$ Fluorescence Images of HA-GO-Treated E. coli

$(\mathrm{S} 35-\mathrm{S} 36)$

Figure S19. LIVE/DEAD ${ }^{\circledR}$ BacLight ${ }^{\mathrm{TM}}$ Fluorescence Images of CGO-Treated $E$. coli

$(\mathrm{S} 37-\mathrm{S} 38)$

Figure S20. LIVE/DEAD ${ }^{\circledR}$ BacLight ${ }^{\mathrm{TM}}$ Fluorescence Images of CG-Treated E. coli

Figure S21. LIVE/DEAD ${ }^{\circledR}$ BacLight ${ }^{\mathrm{TM}}$ Fluorescence Images of HA-CG-Treated $E$. coli

$(\mathrm{S} 41-\mathrm{S} 42)$

Figure S22. LIVE/DEAD ${ }^{\circledR}$ BacLight ${ }^{\mathrm{TM}}$ Fluorescence Images of CCG-Treated E. coli

\section{References}




\section{Supplemental Materials and Methods}

\subsection{Synthetic Methods \\ Graphene Oxide (GO)}

GO was synthesized using a modified Hummer's method, which has been previously reported by this group. ${ }^{1}$ In a $1 \mathrm{~L}$ Erlenmeyer flask, $5 \mathrm{~g}$ of graphite flakes (graphite flake, natural, 325 mesh, 99.8\% metal basis; Alfa Aesar, Ward Hill, MA, USA) were added to $125 \mathrm{~mL}$ of concentrated sulfuric acid (Fisher Scientific, Pittsburgh, PA, USA). The mixture was placed into an ice bath and stirred. Once cooled, $10 \mathrm{~g}$ of $\mathrm{KMnO}_{4}$ (Sigma-Aldrich, St. Louis, MO, USA) was added slowly to the mixture over 20-30 min. Once all the $\mathrm{KMnO}_{4}$ was added, the mixture was removed from the ice bath. After reaching room temperature, the mixture was stirred for $2 \mathrm{~h}$. The mixture was then heated gently to $35^{\circ} \mathrm{C}$ and stirred for another $2 \mathrm{~h}$. The heat was removed, and the reaction was quenched by the addition of $700 \mathrm{~mL}$ of deionized (DI) water, $10 \mathrm{~mL}$ of $30 \%$ hydrogen peroxide (Fisher Scientific), and an additional $225 \mathrm{~mL}$ of DI water. Specifically, the $700 \mathrm{~mL}$ of DI water was added quickly to the reaction in one aliquot to rapidly dissipate the exotherm. Then, $10 \mathrm{~mL}$ of $30 \%$ hydrogen peroxide was added slowly to prevent boil over. The $225 \mathrm{~mL}$ of DI water was also added slowly at first to prevent boil over. When bubbling ceased, the rest of the DI water was rapidly added to the reaction. Then, the reaction was left to stir at room temperature overnight.

GO was purified by vacuum filtrating through a Buchner funnel. The filtrate was discarded, and the filtered GO was carefully removed from the funnel without scrapping the filter paper. Then, the GO was loaded into 3500 molecular weight cutoff dialysis tubing (SnakeSkin ${ }^{\mathrm{TM}}$ dialysis tubing, Thermo Scientific, Waltham, MA, USA) and was dialyzed against $500 \mathrm{~mL}$ of DI water for 3-7 days until the DI water became clear. The DI water was changed twice on the first day of dialysis, and then changed once per day thereafter. After the 3-7 days, the GO was removed from the dialysis tubing and placed into $50 \mathrm{~mL}$ centrifuge tubes, no more than half full. The GO was frozen to $-80^{\circ} \mathrm{C}$ and lyophilized for 3-5 days until dry.

Claisen graphene (CG): CG was synthesized using a previously reported method. ${ }^{1,2}$ In a $1 \mathrm{~L}$, flame dried round bottom flask under nitrogen, $1.94 \mathrm{~g}$ of $\mathrm{GO}$ and $400 \mathrm{~mL}$ of triethyl orthoacetate (Alfa Aesar, Haverhill, MA, USA) was added to the reaction vessel. The reaction was bath sonicated $(240 \mathrm{~W}, 42 \mathrm{kHz}$ ultrasonic cleaner, Kendal) for $10 \mathrm{~min}$ to disperse GO. After sonication, $37.5 \mathrm{mg}$ of $p$-toluene sulfonic acid was added to the mixture while maintaining the reaction under nitrogen. Then, the reaction was refluxed at $142{ }^{\circ} \mathrm{C}$ under nitrogen with stirring for $36 \mathrm{~h}$. The heat was removed, and the reaction was allowed to cool. When the reaction was at $\sim 85^{\circ} \mathrm{C}, 50 \mathrm{~mL}$ of $1.0 \mathrm{M} \mathrm{NaOH}$ (in ethanol) was added, and the stirring was increased to accommodate the increase in the reaction viscosity. When the reaction cooled to room temperature, it was stirred for an additional $3 \mathrm{~h}$.

CG was purified by vacuum filtration using a Buchner funnel. Without scrapping the filter paper, the CG was carefully removed from the funnel and the filtrate discarded. The CG was added to $50 \mathrm{~mL}$ centrifuge tubes, filling no more than $1 / 3$ the volume of the tubes. Then, DI water was added to the $45 \mathrm{~mL}$ mark and $\mathrm{CG}$ was dispersed via vortexing. After dispersing, the CG tubes were centrifuged at 4,200 x g for $5 \mathrm{~min}$. The CG materials separated into three distinct layers. The bottom layer was a solid-black pellet. The layer on top of the pellet was also black but appeared to be a viscous dispersion of graphenic materials. The top layer was an orange colored supernatant. The top two layers were discarded. The CG pellet was washed 3 more times 
with DI water, where centrifugation resulted in only two layers (the CG pellet and the top supernatant that become clearer with each wash). The CG pellet was also washed 2 times with acetone and then dried under vacuum for 24-48 hours until dry.

Hydroxamic acid functional graphenic materials (FGMs): Hydroxamic acid FGMs were prepared with either graphene oxide $(\mathrm{GO})$ or Claisen graphene $(\mathrm{CG})$, generating hydroxamic acid GO (HA-GO) or hydroxamic acid CG (HA-CG), respectively. Briefly, a $250 \mathrm{~mL}$ round bottom flask was charged with $100 \mathrm{mg}$ of either $\mathrm{GO}$ or CG and $100 \mathrm{~mL}$ of dimethyl sulfoxide (Alfa Aesar, Haverhill, MA, USA) under atmospheric conditions. The reaction mixture was sonicated for $10 \mathrm{~min}$ to disperse the graphenic component, followed by the addition of $500 \mathrm{mg}$ of 1-ethyl-3-(3-dimethylaminopropyl)carbodiimide (Oakwood Chemicals, Estill, SC, USA). After $10 \mathrm{~min}$ of stirring at room temperature, $330 \mathrm{mg}$ of N-Hydroxysuccinimide (Chem-Impex International, Wood Dale, IL, USA) was added and stirred for an additional $10 \mathrm{~min}$ at room temperature. Then, $100 \mathrm{mg}$ of hydroxylamine hydrochloride (Sigma-Aldrich, St. Louis, MO, USA) was added using a solution of $500 \mathrm{mg} \mathrm{mL}^{-1}$ of hydroxylamine hydrochloride dissolved in 7.2 M sodium hydroxide (Fisher Scientific, Hampton, NH, USA). The reaction mixture was stirred overnight at room temperature.

To isolate the graphenic component, the reaction was added to $50 \mathrm{~mL}$ centrifuge tubes and centrifuged at 3,600 $\times g$ for 5 min to pellet the graphenic component. The supernatant was discarded. Then, the reaction was purified with a series of wash steps. Deionized (DI) water was added up to the $45 \mathrm{~mL}$ demarcation on the tubes followed by vortexing. The tubes were centrifuged for $5 \mathrm{~min}$ at 3,600 $\times \mathrm{g}$ to re-pellet the graphenic component and separate and discard the supernatant. The washing procedure was repeated with DI water three additional times, followed by two washes with acetone. The graphenic pellet was dried under vacuum for 24-48 hours until dry.

\section{Catechol Functional Graphenic Materials (FGMs):}

Catechol graphene oxide (CGO) and catechol Claisen graphene (CCG) were prepared through an acyl chloride intermediate, which was previously reported. ${ }^{1} \mathrm{In}$ a $100 \mathrm{~mL}$, flame dried round bottom flask under nitrogen, $100 \mathrm{mg}$ of GO or CG, $50 \mathrm{~mL}$ of dry dioxane, and 10 drops of dry dimethylformamide were added. The mixture was bath sonicated for $10 \mathrm{~min}$ to disperse the graphenic component. After sonication, $1.4 \mathrm{~mL}$ of thionyl chloride (Sigma-Aldrich, St. Louis, MO, USA) was slowly added dropwise to the mixture over 5-10 min. The reaction was stirred overnight under nitrogen at room temperature.

The next day, $500 \mathrm{mg}$ of 3,4-dihydroxybenzylamine (DHBA) (Sigma-Aldrich, St. Louis, MO, USA) was added to the round bottom flask while maintaining the reaction under nitrogen. The reaction was heated to $100{ }^{\circ} \mathrm{C}$ and stirred overnight under a nitrogen atmosphere.

The reaction was removed from heat and allowed to cool to room temperature. The reaction was added to $50 \mathrm{~mL}$ centrifuge tubes and centrifuged at 4,200 $\times g$ for $5 \mathrm{~min}$. The graphenic component pelleted and the supernatant, which was orange in color, was discarded. Dichloromethane (DCM) was added to the $45 \mathrm{~mL}$ mark of the centrifuge tubes containing the graphenic pellets and aggressively vortexed. The tubes were centrifuged at 4,200 $\mathrm{g}$ for another 5 min. After centrifugation, the graphenic component floated on the top of the DCM supernatant. The DCM was carefully removed via pipette to prevent loss of the graphenic component and the filtrate discarded. An additional DCM wash was repeated. Then, the graphenic material was washed twice with DI water and twice with acetone, where the graphenic component pelleted on 
the bottom of tubes after centrifugation in both solvents. Then, the graphenic pellets were dried under vacuum for 24-48 hours until dry.

\subsection{Material Characterization}

Fourier Transform Infrared (FTIR) Spectroscopy: FTIR spectra were collected on a PerkinElmer Frontier FT-IR Spectrometer with an attenuated total reflectance (ATR) attachment containing a germanium crystal. Raw spectra were recorded in percent transmittance from 4000$700 \mathrm{~cm}^{-1}$ with a $4 \mathrm{~cm}^{-1}$ resolution. All spectra were processed in Spectrum ${ }^{\mathrm{TM}} 10$ (PerkinElmer) software using an ATR correction, a smooth value of 50, and baseline correction. The automatic baseline correction feature was used as a first pass on spectra followed by a manual baseline correction that used three baseline anchors at 4000, 1900, and $700 \mathrm{~cm}^{-1}$.

Atomic Absorption Spectroscopy (Graphite Furnace): FGMs were dispersed in $2 \mathrm{~mL}$ of a 50 ppm aluminum solution made in a $\mathrm{pH} 4$ nitric acid solution, generating $1 \mathrm{mg} \mathrm{mL}^{-1}$ graphenic dispersions. All samples were agitated for $1 \mathrm{~h}$ on a rotating shaker (MiniMixer ${ }^{\mathrm{TM}}$, Benchmark Scientific, Sayreville, NJ, USA) at room temperature to ensure homogeneity. FGM dispersions were then filtered and the resulting liquids were analyzed using a ThermoFisher iCE 3000 graphite furnace atomic absorption spectrometer. The instrument and autosampler were both set to furnace mode, and the samples were analyzed at a lamp current of 50\% and a wavelength of $394.4 \mathrm{~nm}$. The bandpass was set to $0.2 \mathrm{~mm}$ half-height, the cuvette type was set to ELC (extended life cuvette), and the injection temperature was set to zero. In the event of any solution having an aluminum concentration that did not fit within the calibration curve of 300-700 ppb, the instrument was set to intelligently dilute the outlier sample using a fixed volume of solution to fit the curve.

X-ray Photoelectron Spectroscopy (XPS): XPS analysis was conducted on a Thermo Fisher ESCALAB $250 \mathrm{Xi}$ instrument with an Al K-Alpha source gun and a flood gun in charge compensation standard mode. All spectra were collected using a standard lens mode (angle and field of view of 32,000 steps), Constant Analyzer Energy (CAE) scan mode, and a $200 \mu \mathrm{m}$ spot size. Powdered samples were prepared for XPS analysis using double-sided copper tape as the substrate. Samples were securely adhered to the double-sided copper tape to ensure complete coverage of the substrate without excess loose powder. Survey, C1s, and N1s spectra were collected in triplicate for each sample from three separate spot locations. One Al2p spectrum was collected.

Survey Scans: All survey scan spectra were collected using 5 cumulative scans over a binding energy range of 1350 to $-10 \mathrm{eV}$. Further, all samples were analyzed using a pass energy of 150 $\mathrm{eV}$, an energy step size of $1.0 \mathrm{eV}$, and a dwell time of $10 \mathrm{~ms}$. CasaXPS software (CasaXPS) was used to quantify the elemental composition from survey spectra by integrating the area under peaks unique to each element. Specifically, the O1s (545-525 eV), N1s (410-392 eV), C1s (298$279 \mathrm{eV}), \mathrm{Cl} 2 \mathrm{p}$ (210-190 eV), Br3p (194-172 eV), S2p (175-157 eV), and Al2p (80-68 eV) emission peaks were used to quantify oxygen, nitrogen, carbon, chlorine, bromine, sulfur, and aluminum, respectively. Additionally, a smart background and standard peak types were used for all elemental quantification. For each material, each survey spectrum was collected from separate spot locations to prevent artifacts from sample degradation. 
High Resolution Carbon (C1s) Scans: All high resolution C1s spectra were collected using 10 cumulative scans over a binding energy range of 298 to $279 \mathrm{eV}$. Additionally, all spectra were acquired using a pass energy of $50 \mathrm{eV}$, an energy step size of $0.1 \mathrm{eV}$, and a dwell time of $50 \mathrm{~ms}$. $\mathrm{C} 1 \mathrm{~s}$ spectra were collected from different spot locations to prevent artifacts from sample degradation.

OriginPro 2019b (OriginLab) software was used to smooth raw C1s spectra with the Savitzky-Golay smoothing method. Specifically, the Savitzky-Golay smoothing was carried out using a second-degree polynomial with a 15-point step-size. After smoothing, all spectra were charge corrected to adventitious carbon $(284.8 \mathrm{eV}$ ) and truncated to $292-280 \mathrm{eV}$. Fityk (Version 0.9.8) software was used for Shirley baseline subtraction and Gaussian peak fitting. The peak locations for carboxylic acids $(289.0 \mathrm{eV})$, carbonyls $(287.4 \mathrm{eV})$, alcohols and epoxides (286.5 $\mathrm{eV})$, and $\mathrm{sp}^{2}$ and $\mathrm{sp}^{3}$ hybridized carbon $(284.8 \mathrm{eV})$ were constrained to $\pm 0.2 \mathrm{eV}$. The full width at half maximum (FWHM) for all peaks was constrained to $1.4 \mathrm{eV}$.

High Resolution Nitrogen (N1s) Scans: All high resolution N1s spectra were collected from 410 to $392 \mathrm{eV}$ with 25 cumulative scans per spectrum. Further, spectra were acquired using a pass energy of $50 \mathrm{eV}$, an energy step size of $0.1 \mathrm{eV}$, and a dwell time of $50 \mathrm{~ms}$. Each N1s spectrum was collected at a different spot location on the material to prevent sample degradation due to the $\mathrm{X}$-ray source.

OriginPro 2019b (OriginLab) software was used to smooth raw N1s spectra using the Savitzky-Golay method (second-degree polynomial) with a 25-point step-size. The N1s spectra were truncated to $405-395 \mathrm{eV}$. Fityk (Version 0.9.8) software was then used to remove the Shirley background and deconvolute peaks using Gaussian peak fitting. For catechol FGMs, peak fitting procedures have already been established using appropriate standards. ${ }^{1}$ All peaks were constrained to $\pm 0.2 \mathrm{eV}$ of the peak location with a fixed FWHM. For amines, the peak location was $400.1 \mathrm{eV}$ with a FWHM of $1.64 \mathrm{eV}$. The amide peak location was $398.8 \mathrm{eV}$ with a FWHM of $2.30 \mathrm{eV}$.

High Resolution Aluminum (Al2p) Scans: All high resolution Al2p scans were acquired from 85 to $65 \mathrm{eV}$ with 25 cumulative scans per spectra, a pass energy of $50.0 \mathrm{eV}$, an energy step size of $0.1 \mathrm{eV}$, and a dwell time of $50 \mathrm{~ms}$.

OriginPro 2019b (OriginLab) software was used to smooth raw Al2p spectra with the Savitzky-Golay method (second-degree polynomial) with a 45-point step-size. Spectra were truncated to $80-65 \mathrm{eV}$. Then, spectra were processed in Fityk (Version 0.9.8) software, where the Shirley background was removed. Quantification of the area under the curve of A12p spectra for catechol FGMs (CGO and CCG) was also conducted in Fityk with Gaussian peak fitting. The Al2p spectra have spin-orbit splitting into $2 \mathrm{p} 1$ and $2 \mathrm{p} 3$ peaks; however, the $2 \mathrm{p} 1$ and $2 \mathrm{p} 3$ splitting is closely spaced $(\Delta=0.44 \mathrm{eV})$. CGO and CCG Al2p spectra had two, clearly separated peaks ( $\Delta$ $=\sim 3.0 \mathrm{eV}$ ). Thus, each peak was deconvoluted as one peak without spin-orbit splitting or peak constraints (peak location or FWHM). The peaks in the Al2p spectra for CGO were $70.7 \mathrm{eV}$ $(\mathrm{FWHM}=2.44 \mathrm{eV})$ and $74.6 \mathrm{eV}(\mathrm{FWHM}=1.74 \mathrm{eV})$. The peaks in the Al2p spectra for CCG were $70.2 \mathrm{eV}(\mathrm{FWHM}=2.30 \mathrm{eV})$ and $74.4 \mathrm{eV}(\mathrm{FWHM}=2.32 \mathrm{eV})$. Quantification of each peak was determined from the area under the curve.

Energy-Dispersive X-ray (EDX) Spectroscopy: FGMs were prepared by adhering powders onto double-sided copper tape to generate thick films. EDX was performed using a FEI Quanta 
600. The thick films attached onto conductive copper tape were mounted and imaged at $\times 600$ and $\times 2,500$ magnification under low vacuum with $20 \mathrm{keV}$ incident e-beam. Element mapping was conducted by detecting characteristic X-rays generated from the surface.

Proton Nuclear Magnetic Resonance ( ${ }^{1}$ H-NMR) Spectroscopy: Samples were prepared by dispersing $5 \mathrm{mg}$ of FGMs in $\mathrm{D}_{2} \mathrm{O}$. The dispersions were vigorously vortexed and then filtered through a $0.2 \mu \mathrm{m}$ syringe filter. The filter containing the FGMs was discarded while the filtrate was collected and analyzed via ${ }^{1} \mathrm{H}-\mathrm{NMR}$. A $500 \mathrm{MHz}$ NMR (Bruker AvanceTM 500) was used to acquire spectra with 32 cumulative scans. Spectra were analyzed using MestReNova software (version 14.0.1-23559).

\subsection{FGM Aluminum Chelation}

FGMs were dispersed in $2 \mathrm{~mL}$ of a $50 \mathrm{ppm}$ aluminum solution made in a $\mathrm{pH} 4$ nitric acid solution, generating $1 \mathrm{mg} \mathrm{mL}^{-1}$ graphenic dispersions. All samples were agitated for $1 \mathrm{~h}$ on a rotating shaker (MiniMixer ${ }^{\mathrm{TM}}$, Benchmark Scientific, Sayreville, NJ, USA) at room temperature to ensure homogeneity. FGM dispersions were then filtered. The filtrates were analyzed using the graphite furnace, as described above. The FGMs were collected, frozen at $-80^{\circ} \mathrm{C}$, lyophilized to dryness, and then analyzed via XPS as described above.

\subsection{FGM Regeneration}

To remove aluminum from FGMs, $5 \mathrm{mg}$ of FGMs were dispersed in $5 \mathrm{~mL}$ of a $1.0 \mathrm{M}$ nitric acid solution (at pH 0 ). FGM dispersions were agitated for $1 \mathrm{~h}$ on a rotating shaker (MiniMixer ${ }^{\mathrm{TM}}$, Benchmark Scientific, Sayreville, NJ, USA) at room temperature. The FGM dispersions were then centrifuged at $4,200 \times g$ for $5 \mathrm{~min}$ and the supernatant was discarded. The FGMs were collected, frozen at $-80^{\circ} \mathrm{C}$, and lyophilized to dryness. FGMs were then analyzed via XPS as described above.

\subsection{FGM Stability in Acidic Water}

A total of $5 \mathrm{mg}$ of FGMs were dispersed in $1 \mathrm{~mL}$ of a $1.0 \times 10^{-4} \mathrm{M}$ nitric acid solution (at $\mathrm{pH} 4$ ). After one week at room temperature, the tubes were centrifuged at $4,200 \times g$ for $5 \mathrm{~min}$ and the supernatant was discarded. The materials were washed with fresh acetone and centrifuged at $4,200 \times g$ for $5 \mathrm{~min}$. The supernatant was discarded and the FGMs were washed with fresh acetone one additional time, centrifuged, and the supernatant was discarded. Then, the FGMs were dried under vacuum and analyzed via XPS as described above.

\subsection{FGM Bacterial Culture}

Aseptic technique was utilized when preparing and handling all samples to prevent contamination.

Buffered Media Preparation: Media was prepared using a modified method, where $12.5 \mathrm{~g}$ of LB Miller Broth (Fisher BioReagents ${ }^{\mathrm{TM}}$, USA) and $0.75 \mathrm{~g}$ of Tris $\mathrm{HCl}$ (Promega, Madison, WI, USA) were dissolved in $500 \mathrm{~mL}$ of deionized water to generate a final media concentration of 10 $\mathrm{g} \mathrm{L}^{-1}$ tryptone, $10 \mathrm{~g} \mathrm{~L}^{-1} \mathrm{NaCl}$, and $5 \mathrm{~g} \mathrm{~L}^{-1}$ yeast extract. Media was then sterilized at $121{ }^{\circ} \mathrm{C}$ for 1 $\mathrm{h}$ in an autoclave. Media was cooled to room temperature before use. 
Bacterial Culture: Escherichia coli (E. coli) strain K12 was purchased from ATCC (ATCC® $25404^{\mathrm{TM}}$ ). Cultures were maintained in $5 \mathrm{~mL}$ of buffered media in a $50 \mathrm{~mL}$ centrifuge tube with a loosened cap, on a rotating shaker (MiniMixer ${ }^{\mathrm{TM}}$, Benchmark Scientific, Sayreville, NJ, USA) at $37^{\circ} \mathrm{C}$ (MyTemp Mini Digital Incubator, Benchmark Scientific). Cultures were propagated for $16 \mathrm{~h}$ at $37^{\circ} \mathrm{C}$, generating a cell concentration of $\sim 2 \times 10^{9}$ bacteria $\mathrm{mL}^{-1}$. Then, bacteria were subcultured by centrifugation at $10,000 \times g$ for $15 \mathrm{~min}$ to pellet the cells, followed by aspiration of the supernatant and resuspension of the pellet in $5 \mathrm{~mL}$ of fresh, buffered media. Cultures were then used for experiments in a 1:4 split ratio with fresh, buffered media $(1 \mathrm{~mL}$ stock cell suspension : $4 \mathrm{~mL}$ fresh, buffered media).

FGM Stock Dispersions: FGMs were weighed into $20 \mathrm{~mL}$ glass scintillation vials and irradiated with $254 \mathrm{~nm}$ ultraviolet light for $5 \mathrm{~min}$ for sterilization. Powders were dispersed in buffered media (sterilized, buffered LB Miller Broth) to a final concentration of $2.00 \mathrm{mg} \mathrm{mL}^{-1}$. Then, the FGM $2.00 \mathrm{mg} \mathrm{mL}^{-1}$ stock dispersions were serially diluted to generate two additional FGM stock dispersions of 0.20 and $0.02 \mathrm{mg} \mathrm{mL}^{-1}$ for further testing.

LIVE/DEAD ${ }^{\circledR}$ BacLight ${ }^{T M}$ Assay: All samples were run in triplicate, except for the negative control, where five negative control samples were run to ensure enough bacterial culture was available to generate a calibration curve with the LIVE/DEAD ${ }^{\circledR}$ BacLight ${ }^{\mathrm{TM}}$ Bacterial Viability Kit (ThermoFisher Scientific, Waltham, MA, USA). Samples (containing $250 \mu \mathrm{L}$ per well) were prepared in the interior wells of a 96-well cell culture plate to prevent evaporation over the course of the experiment. All samples contained 4\% v/v E. coli from the $1: 4$ split ratio of the bacterial stock. Negative controls and positive controls were run in conjunction with the FGM samples. The negative controls were not treated and only contained $E$. coli and media while the positive controls were dosed with Penicillin/Streptomycin diluted to $100 \mathrm{U} \mathrm{mL}^{-1}$ (ThermoFisher Scientific). The FGM samples were prepared by dosing E. coli with FGM stock dispersions $\left(2.00,0.20,0.02 \mathrm{mg} \mathrm{mL}^{-1}\right)$ to final concentrations of either $1.00,0.10$, or $0.01 \mathrm{mg} \mathrm{mL}^{-1}$. Finally, the 96 -well cell culture plate (with cell culture plate lid) was incubated at $37^{\circ} \mathrm{C}$ on a rotational shaker for $16 \mathrm{~h}$.

After a $16 \mathrm{~h}$ incubation, the 96-well culture plate was removed from the rotational shaker and the $37{ }^{\circ} \mathrm{C}$ incubator. The plate was placed on a flat surface and undisturbed for $15 \mathrm{~min}$ at room temperature to ensure FGMs settled to the bottom of wells. Then, $125 \mu \mathrm{L}$ was removed from the top of each well (with care taken to leave FGMs on the bottom of the wells) and used for the LIVE/DEAD ${ }^{\circledR}$ BacLight ${ }^{\mathrm{TM}}$ assay.

A modified procedure for the microplate LIVE/DEAD ${ }^{\circledR}$ BacLight ${ }^{\mathrm{TM}}$ assay was used. ${ }^{5}$ That is, the $125 \mu \mathrm{L}$ aliquots obtained from the 96-well culture plate were centrifuged (Eppendorf Microcentrifuge Model 5430) at 10,000 $\times \mathrm{g}$ for $10 \mathrm{~min}$ to pellet bacteria. The pelleted bacteria were an opaque white and visually did not contain black specks indicative of FGMs. The supernatant consisting of media was carefully removed without disturbing the pelleted bacteria. Bacterial pellets were gently re-dispersed in $1.0 \mathrm{~mL}$ of $0.85 \% \mathrm{NaCl}$ buffer for all samples except two of the negative control samples. The remaining two negative control samples were used to generate the calibration curve. One negative control sample was re-dispersed in $1.5 \mathrm{~mL}$ of $0.85 \%$ $\mathrm{NaCl}$ buffer and designated the $100 \%$ live sample. The second negative control sample was 
designated as the $0 \%$ live sample and was dispersed in $0.5 \mathrm{~mL}$ of $0.85 \% \mathrm{NaCl}$ buffer and $1.0 \mathrm{~mL}$ of $70 \%$ isopropyl alcohol. The $100 \%$ and $0 \%$ live sample were incubated with loosened lids for 1 $\mathrm{h}$ at room temperature and inverted every $15 \mathrm{~min}$. After the $1 \mathrm{~h}$ incubation, the samples were centrifuged at $10,000 \times g$ for $10 \mathrm{~min}$, the supernatant discarded, and bacterial pellets were carefully re-dispersed in $1.0 \mathrm{~mL}$ of $0.85 \% \mathrm{NaCl}$ buffer. The $100 \%$ and $0 \%$ live were then used to create $90 \%, 50 \%$, and $10 \%$ live samples for the calibration curve.

After all samples were prepared and dispersed in $0.85 \% \mathrm{NaCl}$ buffer, $100 \mu \mathrm{L}$ of each sample was loaded into a 96-well culture plate. Absorbance at $670 \mathrm{~nm}$ was acquired on a Spark ${ }^{\circledR}$ plate reader (Tecan) with SparkControl ${ }^{\mathrm{TM}}$ v2.2 software. Samples were diluted with $0.85 \% \mathrm{NaCl}$ buffer accordingly to reach an optical density of 0.06 at $670 \mathrm{~nm}$.

The LIVE/DEAD ${ }^{\circledR}$ BacLight ${ }^{\mathrm{TM}}$ stain was prepared by dispersing $3 \mu \mathrm{L}$ of Syto ${ }^{\circledR} 9$ and 3 $\mu \mathrm{L}$ of propidium iodide for every $2 \mathrm{~mL}$ of deionized water. Then, $100 \mu \mathrm{L}$ of stain was added into each well of the 96 -well culture plate containing $100 \mu \mathrm{L}$ of sample. The plate was protected from light and incubated for $15 \mathrm{~min}$ at room temperature.

Fluorescence spectra for all samples stained with the LIVE/DEAD ${ }^{\circledR}$ BacLight ${ }^{\mathrm{TM}}$ assay were collected on a Spark ${ }^{\circledR}$ plate reader with SparkControl ${ }^{\mathrm{TM}}$ v2.2 software. All spectra were acquired with a manual gain of 60 from a z-position of $17,152 \mu \mathrm{m}$, an excitation wavelength of $485 \mathrm{~nm}$ with a bandwidth of $10 \mathrm{~nm}$, and an emission spectrum from 500-700 nm with a bandwidth of $10 \mathrm{~nm}$ and $5 \mathrm{~nm}$ step size.

Fluorescence microscopy samples were collected using an EVOS ${ }^{\circledR}$ FL Auto Cell Imaging System (ThermoFisher Scientific) with a $100 \times, 1.40$ numerical aperture, oil-immersion objective. Samples were prepared by depositing $10 \mu \mathrm{L}$ of stained bacteria onto a $25 \times 75 \mathrm{~mm}, 1.0$ $\mathrm{mm}$ thick microscope slide. After depositing bacterial samples, samples were left for 2-5 minutes on a flat surface to allow bacteria to settle onto the microscope slide for co-localization fluorescence imaging.

LIVE/DEAD B BacLight ${ }^{T M}$ Assay FGM Control Experiment: To ensure that FGMs did not interfere with the LIVE/DEAD ${ }^{\circledR}$ BacLight ${ }^{\mathrm{TM}}$ assay, a control experiment was run. Six no treatment samples were cultured in a 96-well cell culture plate using the same procedure as described above. For each sample, $125 \mu \mathrm{L}$ was removed from each well and centrifuged at $10,000 \times g$ for $10 \mathrm{~min}$ to pellet bacteria. The buffered media was removed with care taken not to disturb the bacterial pellets. The pellets were then re-dispersed in $1.0 \mathrm{~mL} 0.85 \% \mathrm{NaCl}$ buffer containing either $1.0,0.10,0.010,0.0010,0.00010$, or $0.0 \mathrm{mg} \mathrm{mL}^{-1}$ of GO or CG. Then, $100 \mu \mathrm{L}$ of samples were added to a 96-well plate and absorbance was measured from 280 to $800 \mathrm{~nm}$ with a step size of $1 \mathrm{~nm}$. Further, samples were stained using the LIVE/DEAD® BacLight ${ }^{\mathrm{TM}}$ assay as described above and fluorescence emission spectra were acquired. An additional step prior to measuring fluorescence was added to ensure that FGMs were properly dispersed. That is, a 20 second orbital shaking step with an amplitude of $1.0 \mathrm{~mm}$ and frequency of $510 \mathrm{rpm}$ was performed on the 96-well plate in the Spark® plate reader. 


\section{Supplemental Results and Discussion}

\subsection{Hydroxamic Acid Functionalized FGMs}

$\mathrm{X}$-ray photoelectron spectroscopy (XPS) showed that hydroxamic acid graphene oxide (HA-GO) and hydroxamic acid Claisen graphene (HA-CG) contained nitrogen, which is a unique element of hydroxamic acids. However, reagents used to synthesize HA-GO and HA-CG and the resulting byproducts of the reaction also contain nitrogen. For example, 1-Ethyl-3-(3dimethylaminopropyl)carbodiimide (EDC), N-hydroxysuccinimide (NHS), and hydroxylamine $\left(\mathrm{NH}_{2} \mathrm{OH}\right)$ were reagents necessary to synthesize HA-GO and HA-CG and all contain nitrogen. Further, the urea byproduct from the carbodiimide coupling also contains nitrogen. Thus, XPS high resolution N1s analysis and Fourier transform infrared (FTIR) spectroscopy were utilized to try to identify the chemical state of the nitrogen signal in the HA-GO and HA-CG materials. Unfortunately, the hydroxamic acid moieties in HA-GO and HA-CG did not produce a unique peak in the XPS high resolution N1s spectra. Further, the hydroxamic acid stretch $\left(1650 \mathrm{~cm}^{-1}\right)$, which was determined using benzohydroxamic acid as a standard, overlapped with other stretches from the graphenic backbones (Figure S4). Instead, we were able to use a variety of analytical techniques to show that HA-GO and HA-CG did not contain any residual reagents or byproducts from the synthesis, suggesting the nitrogen content was a result of hydroxamic acids.

FTIR spectroscopy was utilized to determine the presence of impurities (either reagents or byproducts from synthesis) in HA-GO and HA-CG. As shown in Figure S4, HA-GO and HA$\mathrm{CG}$ did not contain the carbodiimide (CDI), imide, or $\mathrm{NH}_{2} \mathrm{OH}$ (denoted as *) stretches, indicative of the presence of EDC, NHS, and $\mathrm{NH}_{2} \mathrm{OH}$, respectively. A model urea compound did not contain any unique stretches in the FTIR spectra.

Proton nuclear magnetic resonance ( $\left({ }^{1} \mathrm{H}-\mathrm{NMR}\right)$ spectroscopy was utilized to confirm that the urea byproduct was not contributing to the nitrogen signal in the HA-GO and HA-CG materials (Figure S5). All of the nitrogen-containing reagents and byproducts are water soluble. Thus, FGMs were dispersed in $\mathrm{D}_{2} \mathrm{O}$ and vigorously mixed to remove any water-soluble impurities from the graphenic backbones. The graphenic component was removed via filtration and the filtrate containing any water-soluble impurities was analyzed. The ${ }^{1} \mathrm{H}-\mathrm{NMRspectra}$ show that there was no measurable urea byproduct, EDC, or NHS present in HA-GO or HA-CG samples.

\subsection{Catechol Functionalized FGMs}

Our catechol FGMs contained some impurities from the synthetic method (sulfur, chlorine, and bromine) (Figure S2), an observation we have previously reported with our materials. ${ }^{1}$ Impurities can adversely affect bacterial compatibility. However, we demonstrated that our FGMs are well tolerated by E. coli even with high concentrations of catechol FGMs (up to $1.0 \mathrm{mg} \mathrm{mL}^{-1}$ ) (Figures S19 and S22). This observation contrasts what we have previously reported for the catechol FGMs, where previous work has demonstrated moderate antimicrobial capacity. However, there were significantly less impurities in the FGMs utilized for this study, which may explain the enhanced tolerance of $E$. coli cultures with catechol FGMs.

\subsection{Chelation Capacity and Regeneration of FGMs}

After 1 week in acidic conditions that mimic the environment at an acid mine drainage site $(\mathrm{pH} 4)$, we confirmed that the chelating groups on the graphenic backbones of FGMs remained unchanged. There were negligible changes in the XPS elemental composition (Figure 
S11), carboxylic acids (Figure S12), or amide bound catechols (Figure S13) of the FGMs when compared to the unaged FGM powders (Figures S2, S3, S6).

\subsection{Ecological Impact of FGMs}

The compatibility of $E$. coli cells was evaluated to ascertain the effect our FGMs may have on microflora in the environment. The LIVE/DEAD ${ }^{\circledR}$ BacLight $^{\mathrm{TM}}$ assay was used for these cell studies, where the assay utilizes two fluorescent probes to determine the percent of live $E$. coli cells. FGMs are notorious fluorophore quenchers and have been documented to interfere with fluorescent assays such as the methylthiazolyldiphenyl-tetrazolium bromide (MTT) assay for mammalian cells. ${ }^{3}$ Thus, we performed a control experiment with the LIVE/DEAD ${ }^{\circledR}$ BacLight $^{\mathrm{TM}}$ assay to ascertain if our FGMs quenched the fluorophores in the assay.

No treatment samples only containing $E$. coli cells were utilized for the control experiment. Then, either GO or CG was added to the cells. The total concentrations of GO and CG spanned several orders of magnitude $\left(0.0,0.0001,0.001,0.01,0.1\right.$, and $\left.1.0 \mathrm{mg} \mathrm{mL}^{-1}\right)$.

We analyzed the absorbance of the samples and found that our FGM materials cause minimal assay interference. The GO and CG samples were compared to a sample that was not treated with FGMs. We found that GO only began to interfere with the absorbance of the assay at very high concentrations. $\mathrm{CG}$, on the other hand did not display any deviations from the no treatment control (Figure S15).

The samples were then subjected to the LIVE/DEAD® ${ }^{B}$ BacLight $^{\mathrm{TM}}$ assay. The green-tored fluorescence ratio also demonstrated that $\mathrm{GO}$ and $\mathrm{CG}$ did not interfere with the assay in comparison to a no treatment control (Figure S16).

Based on the control experiment results, we concluded that our FGMs did not interfere with the results of the LIVE/DEAD ${ }^{\circledR}$ BacLight ${ }^{\mathrm{TM}}$ assay utilized to measure viability of experimental samples. Extreme care was taken to remove FGMs from samples prior to testing (see SI Materials and Methods). Thus, only very low concentrations of FGMs were present in our experimental samples, where FGM concentrations were much lower than those that interfere with the assay. 


\section{Supplemental Reaction Schemes}

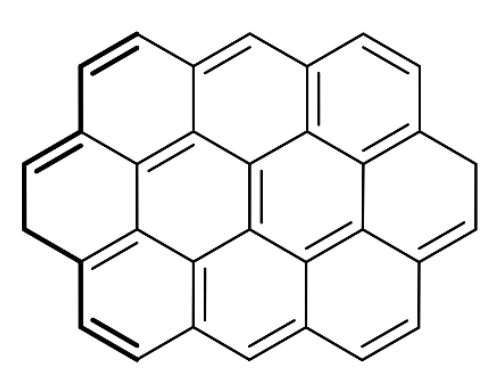

Graphite
$\underset{\mathrm{H}_{2} \mathrm{O}, \mathrm{H}_{2} \mathrm{O}_{2}}{\stackrel{\mathrm{SO}_{4}, \mathrm{KMnO}_{4}}{\longrightarrow}}$

Scheme S1. Synthesis of graphene oxide (GO) from graphite using a modified Hummers' method. Note that a section of a graphenic flake is represented for simplicity, where bold lines represent sheet edges. 


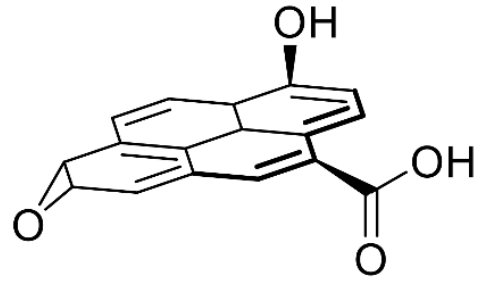

Graphene Oxide (GO)

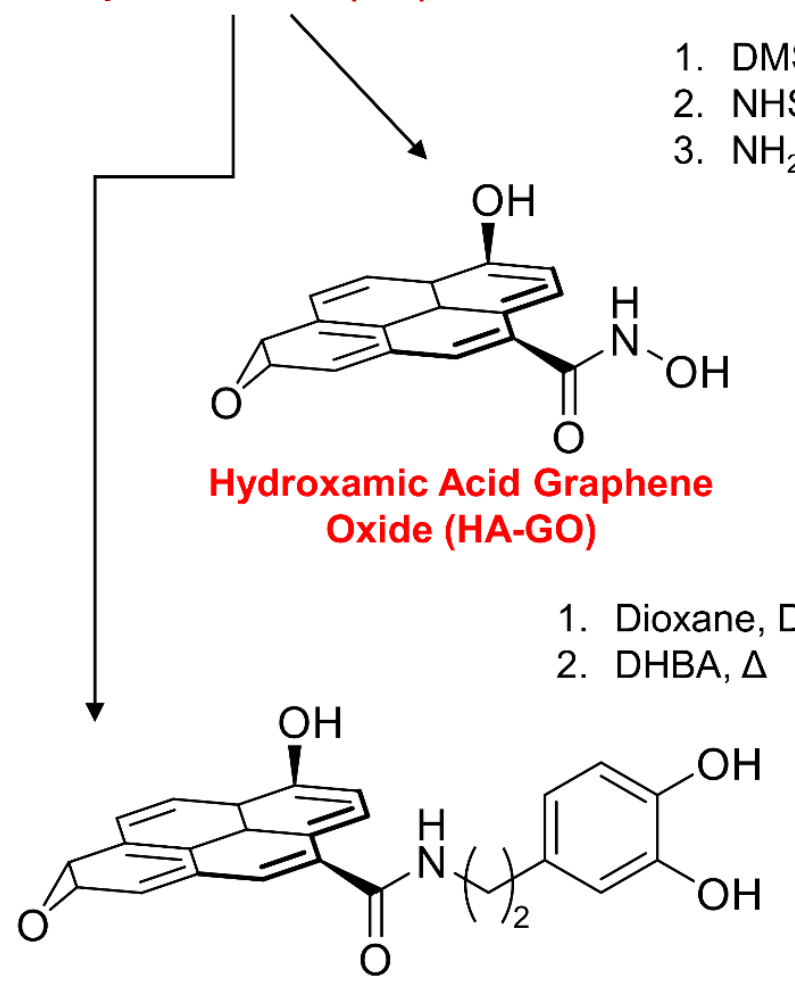

Catechol Graphene Oxide (CGO)

2. DHBA, $\Delta$
1. TEOA, TsOH, $\triangle$

2. $1.0 \mathrm{M} \mathrm{NaOH}, \mathrm{RT}$

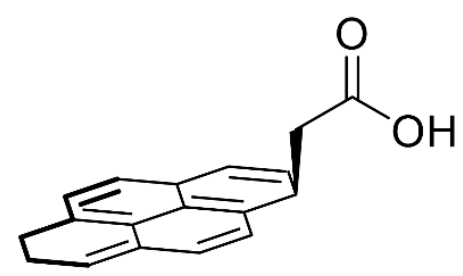

Claisen Graphene (CG)
Hydroxamic Acid Claisen

Graphene (HA-CG)

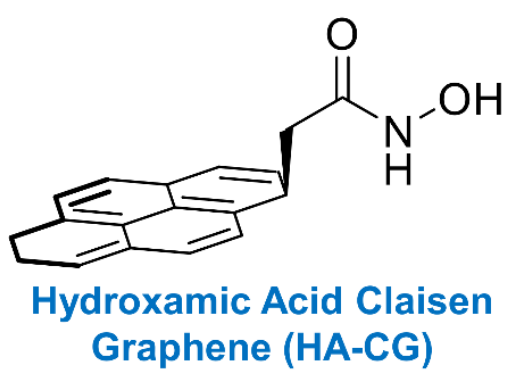

1. Dioxane, DMF, $\mathrm{SOCl}_{2}, \mathrm{RT}$

Scheme S2. Synthetic scheme to generate Claisen graphene (CG), hydroxamic acid functional graphenic materials (FGMs), and catechol FGMs. CG was synthesized from GO using a modified, Johnson-Claisen reaction. Hydroxamic acid FGMs were prepared from either GO or CG using a carbodiimide-assisted procedure with hydroxylamine, generating either hydroxamic acid graphene oxide (HA-GO) or hydroxamic acid Claisen graphene (HA-CG). Lastly, catechol FGMs were synthesized from either GO or CG via thionyl chloride amidation of FGMs with 3,4dihydroxybenzylamine (DHBA). The resulting catechol FGMs were catechol graphene oxide (CGO) and catechol Claisen graphene (CCG). Note that graphenic flakes are represented as pyrene structures for simplicity and bold lines signify sheet edges. 


\section{Supplemental Figures}
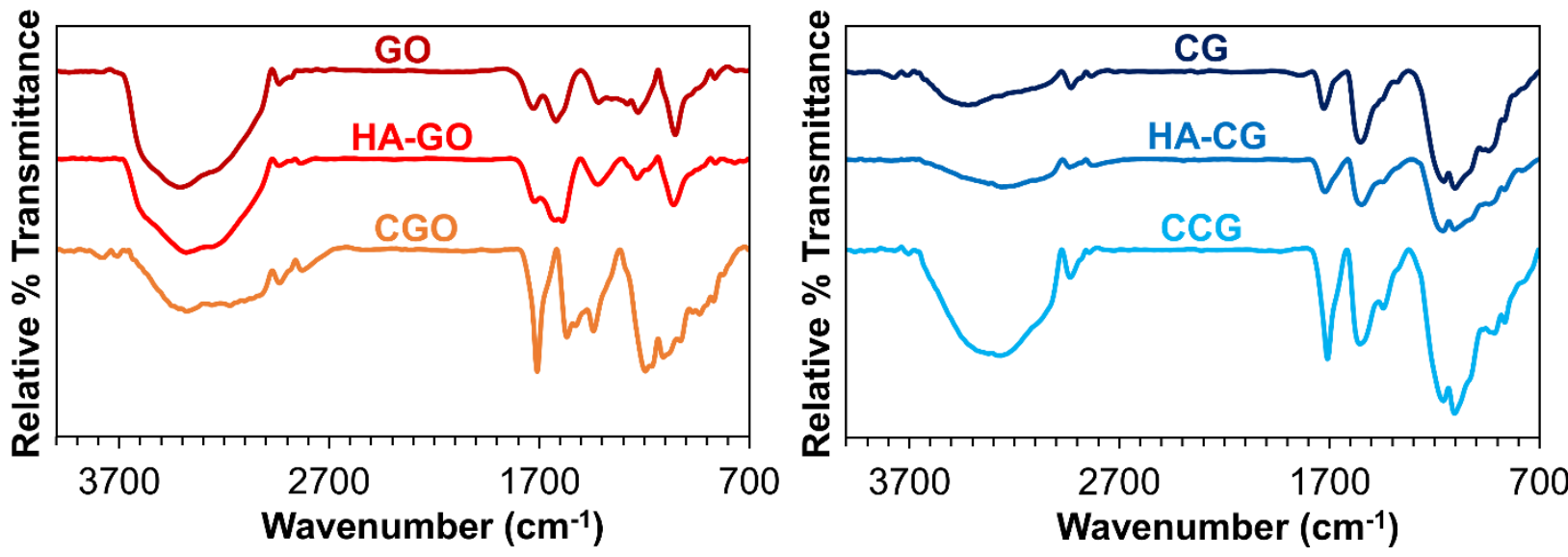

Figure S1. Fourier transform infrared (FTIR) spectroscopy of functional graphenic materials (FGMs). Edge functionalized FGMs containing carboxylic acids (graphene oxide, GO), hydroxamic acid graphene oxide (HA-GO), and catechol graphene oxide (CGO) are shown in dark red, red, and orange, respectively. Basal plane functionalized FGMs containing carboxylic acids (Claisen graphene, CG), hydroxamic acid Claisen graphene (HA-CG), and catechol Claisen graphene (CCG) are shown in dark blue, blue, and light blue, respectively. All spectra were offset for clarity. 
A
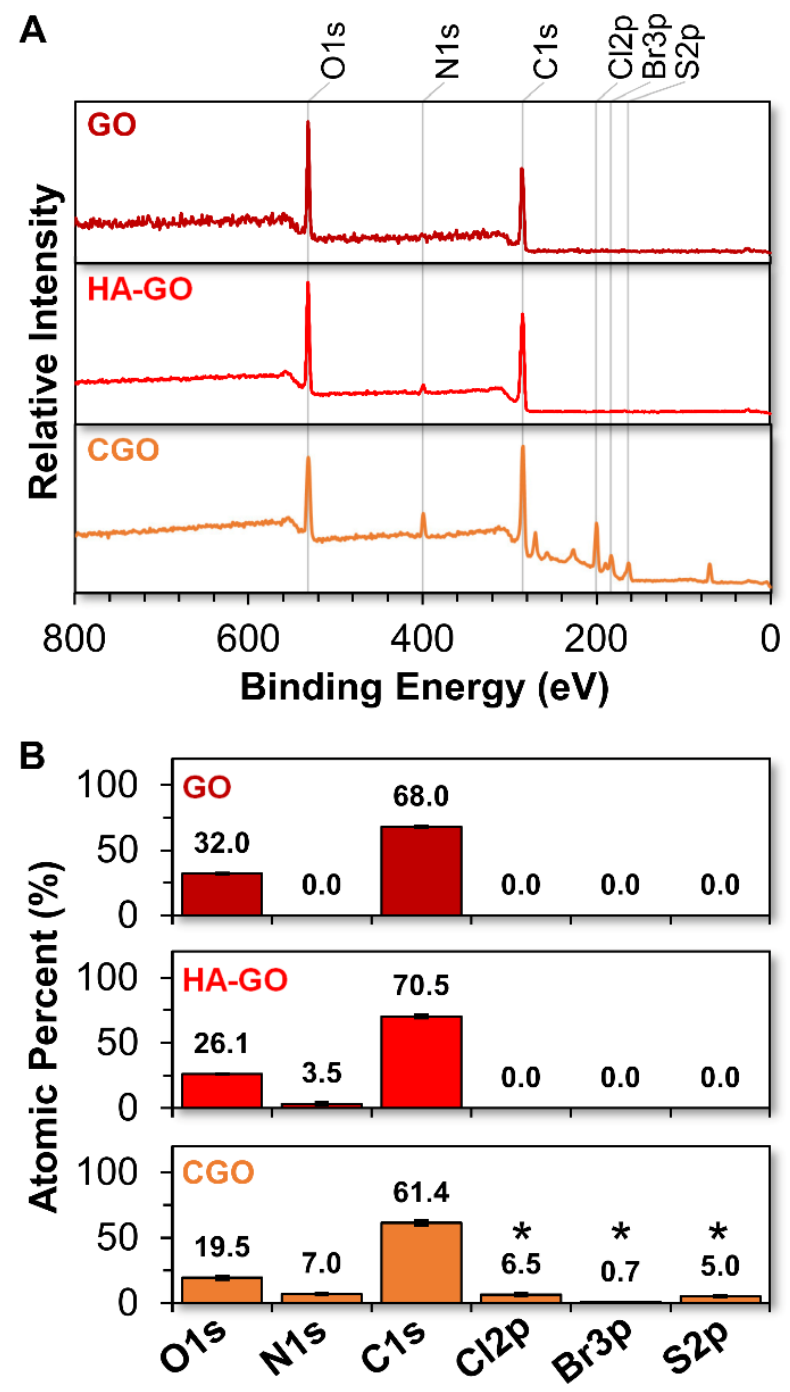

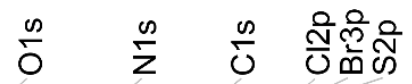
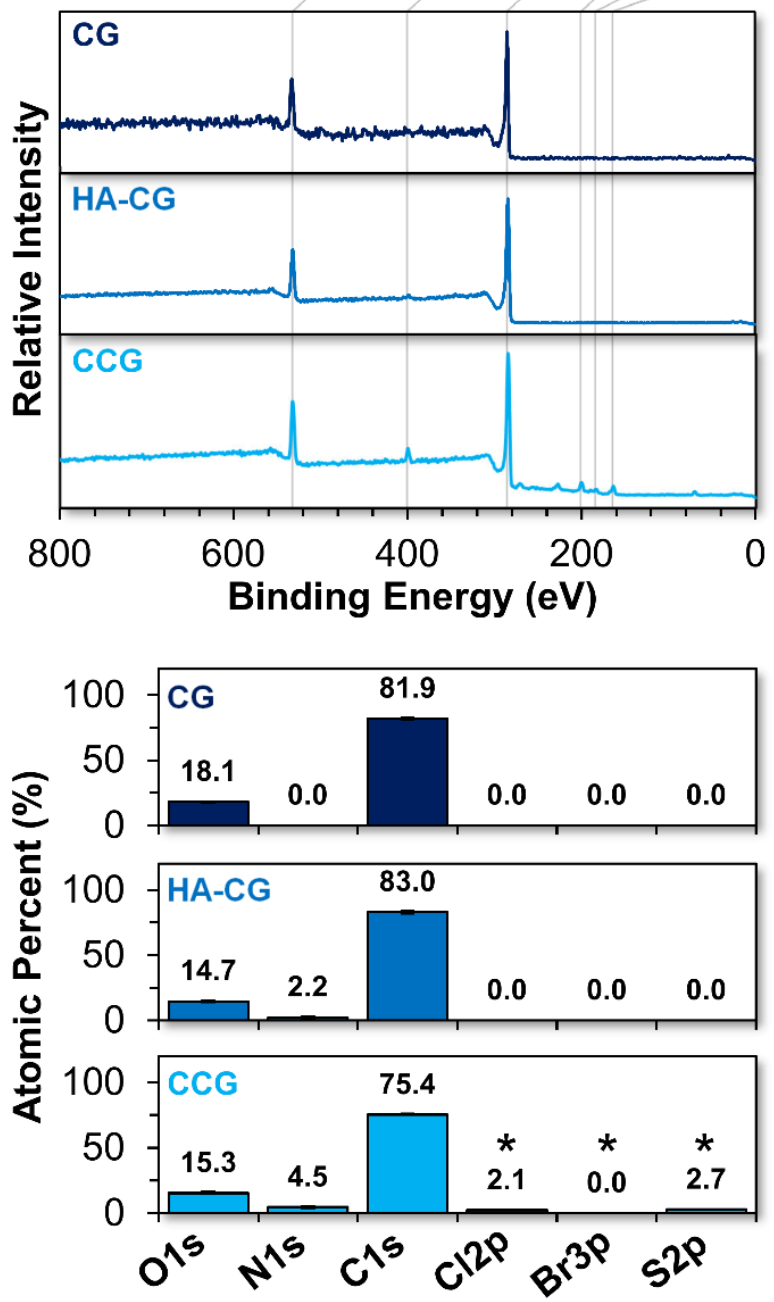

Figure S2. X-ray photoelectron spectroscopy (XPS) of edge- and basal plane-modified functional graphenic material (FGM) powders. Edge functionalized FGMs containing carboxylic acids (graphene oxide, GO), hydroxamic acid graphene oxide (HA-GO), and catechol graphene oxide (CGO) are shown in dark red, red, and orange, respectively. Basal plane functionalized FGMs containing carboxylic acids (Claisen graphene, CG), hydroxamic acid Claisen graphene (HA-CG), and catechol Claisen graphene (CCG) are shown in dark blue, blue, and light blue, respectively. A) XPS survey spectra of FGMs with the oxygen (O1s), nitrogen (N1s), carbon $(\mathrm{C} 1 \mathrm{~s})$, chlorine $(\mathrm{Cl} 2 \mathrm{p})$, bromine $(\mathrm{Br} 3 \mathrm{p})$, and sulfur $(\mathrm{S} 2 \mathrm{p})$ emission peaks labelled. B) Elemental composition of FGMs using the emission peaks labelled in Panel A). Bars represent the average of $n=3$ measurements and the error bars are the standard deviation. Note that chlorine, bromine, and sulfur are denoted with asterisks because they are impurities resulting from the synthesis pathway of CGO and CCG. 
A

Carboxylic Acid

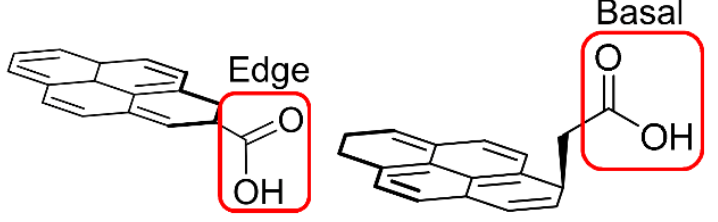

\section{Carbonyl}

Alcohol \&

Epoxide

$\mathrm{sp}^{3} \& \mathrm{sp}^{2}$

Carbon
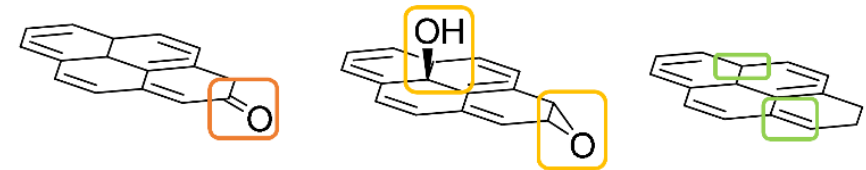

B

Graphene Oxide

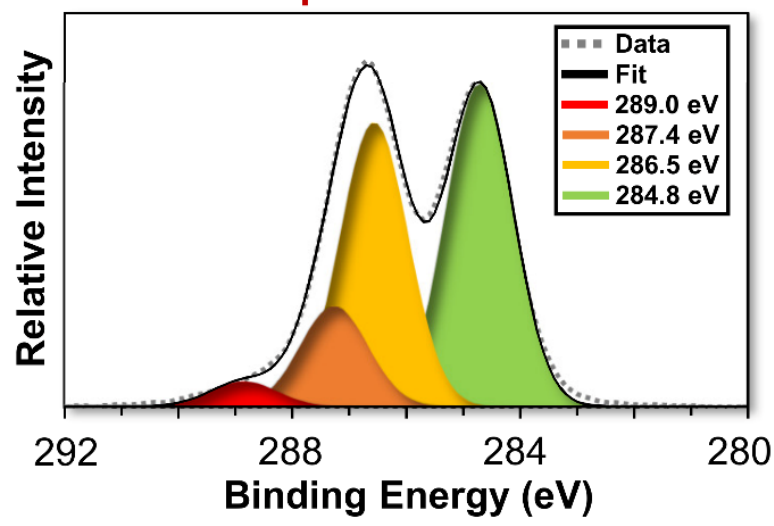

Claisen Graphene
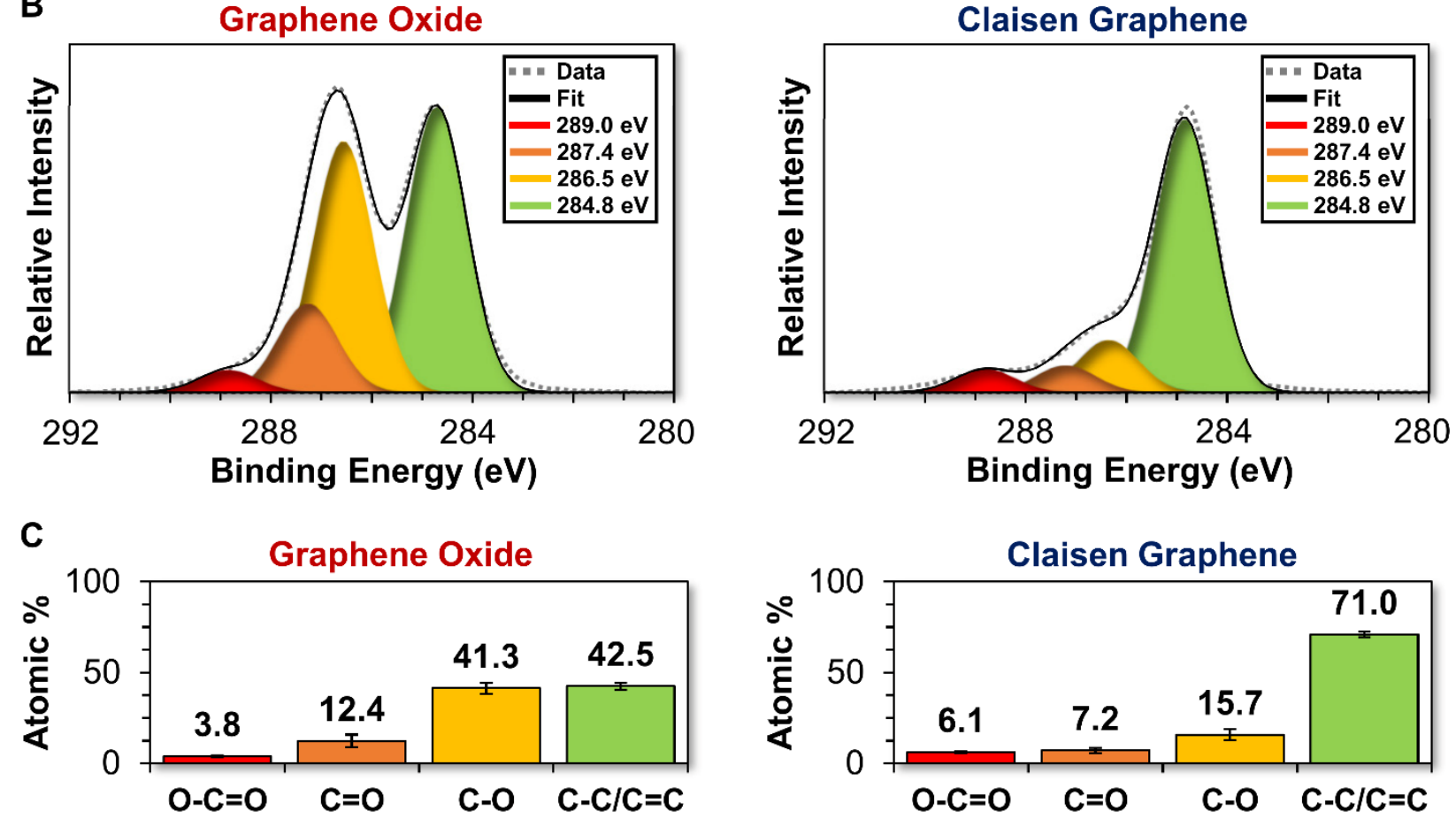

Figure S3. Deconvolution of high-resolution X-ray photoelectron spectroscopy (XPS) carbon (C1s) spectra of edge- and basal plane-modified functional graphenic material (FGM) powders. Edge functionalized FGMs containing carboxylic acids (graphene oxide, GO) and basal plane functionalized FGMs containing carboxylic acids (Claisen graphene, CG) are shown in dark red and dark blue, respectively. A) Representation of the carbon-containing functional groups on the FGMs, where carboxylic acids are red, carbonyl groups are dark orange, alcohol and epoxides are light orange, and $\mathrm{sp}^{3}$ and $\mathrm{sp}^{2}$ carbons are light green. Note that edge and basal plane carboxylic acids are indistinguishable in the high-resolution spectra. B) Deconvoluted XPS C1s spectra of GO and CG. Emission peaks for each type of carbon functional group is color coded to correspond to Panel A). C) Quantification of the atomic percent of carbon groups in GO and CG, determined by taking the area under the curve of each emission peak in Panel B). Bars represent the average of $n=3$ measurements and the error bars are the standard deviation. 


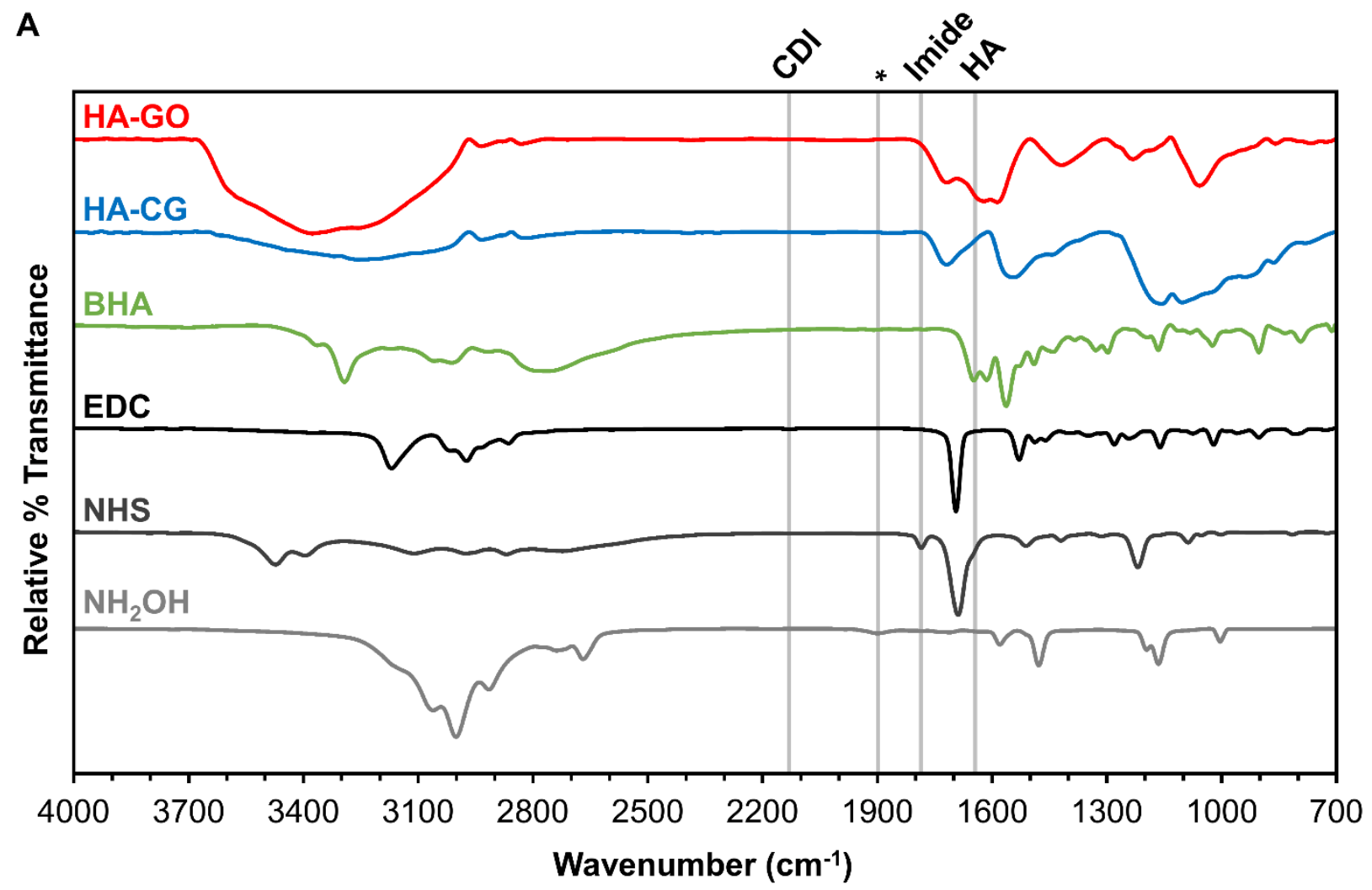

B
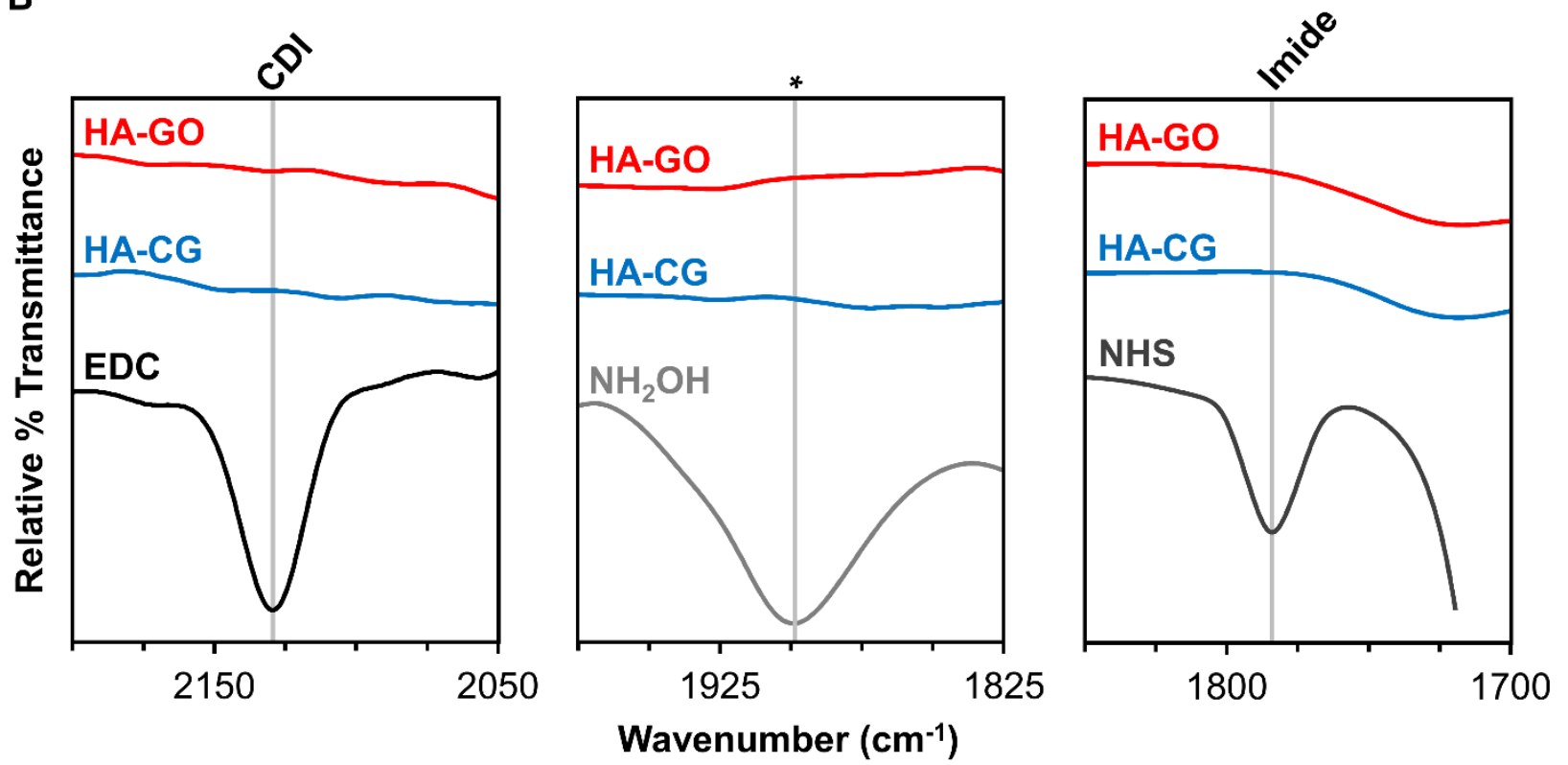

Figure S4. Fourier transform infrared (FTIR) spectroscopy of hydroxamic acid functional graphenic materials (FGMs) and the reagents used and byproducts produced during synthesis. A) FTIR spectra of hydroxamic acid graphene oxide (HA-GO), hydroxamic acid Claisen graphene (HA-CG), benzohydroxamic acid (BHA), 1-Ethyl-3-(3-dimethylaminopropyl)carbodiimide (EDC), N-hydroxysuccinimide (NHS), and hydroxylamine $\left(\mathrm{NH}_{2} \mathrm{OH}\right)$. The carbodiimide (CDI), imide, and hydroxamic acid (HA) stretches indicative of EDC, NHS, and the hydroxamic acid 
functional group on BHA, respectively, are labelled. Further, a peak indicative of $\mathrm{NH}_{2} \mathrm{OH}$ is indicated by an *. B) Inset of FTIR spectra from panel A, where the CDI, *, and imide stretches are labelled, indicating that $\mathrm{EDC}, \mathrm{NH}_{2} \mathrm{OH}$, and $\mathrm{NHS}$ are not present in the hydroxamic acid FGMs. 

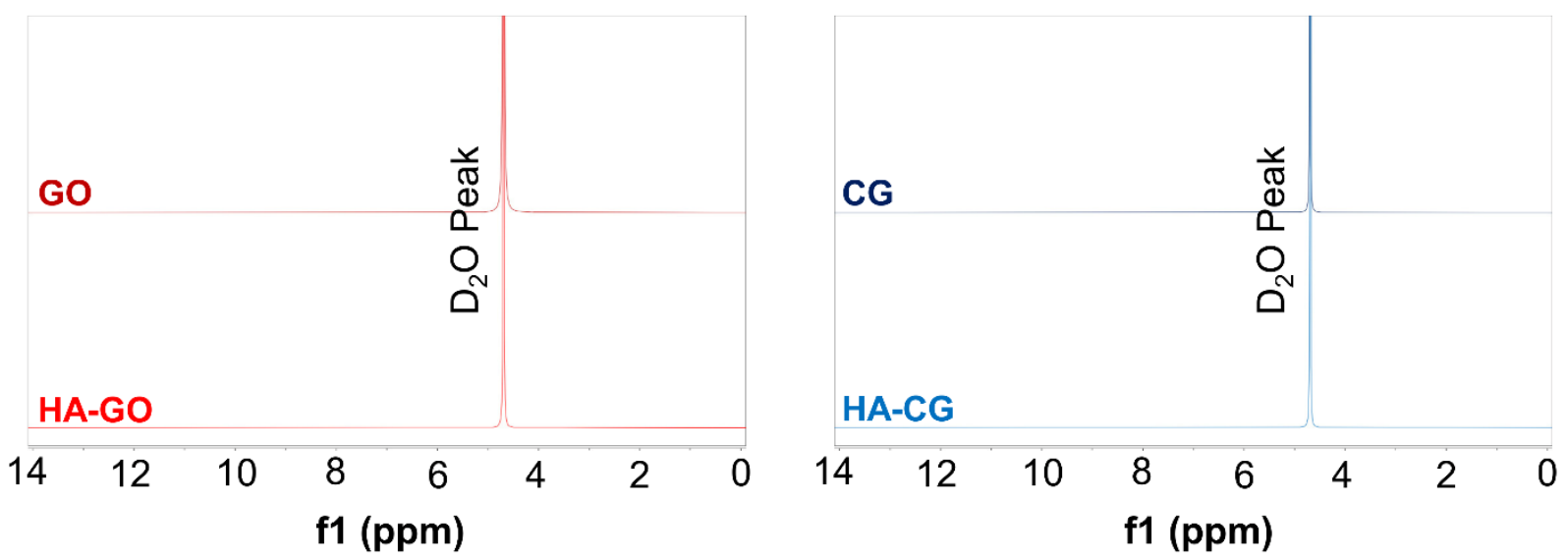

Figure S5. Proton nuclear magnetic resonance $\left({ }^{1} \mathrm{H}-\mathrm{NMR}\right)$ spectroscopy of functional graphenic material (FGM) $\mathrm{D}_{2} \mathrm{O}$ washes. Graphene oxide (GO), hydroxamic acid graphene oxide (HA-GO), Claisen graphene (CG), and hydroxamic acid Claisen graphene (HA-CG) sample spectra are shown in dark red, red, dark blue, and blue, respectively. The FGMs were filtered to separate the FGM and $\mathrm{D}_{2} \mathrm{O}$ filtrate. The ${ }^{1} \mathrm{H}$-NMR spectra were acquired using the $\mathrm{D}_{2} \mathrm{O}$ filtrates, where the $\mathrm{D} 2 \mathrm{O}$ solvent peaks at $4.8 \mathrm{ppm}$ are labelled in the spectra. 


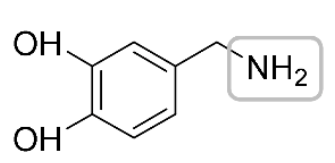<smiles>CC(=O)Oc1ccc(CN)cc1O</smiles>

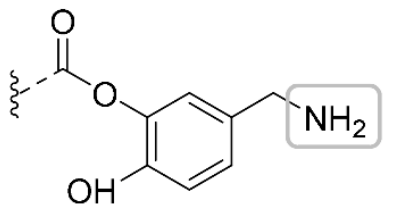<smiles>O=C1C2CCC(C2)N1c1ccc(O)c(O)c1</smiles>

B Catechol Graphene Oxide
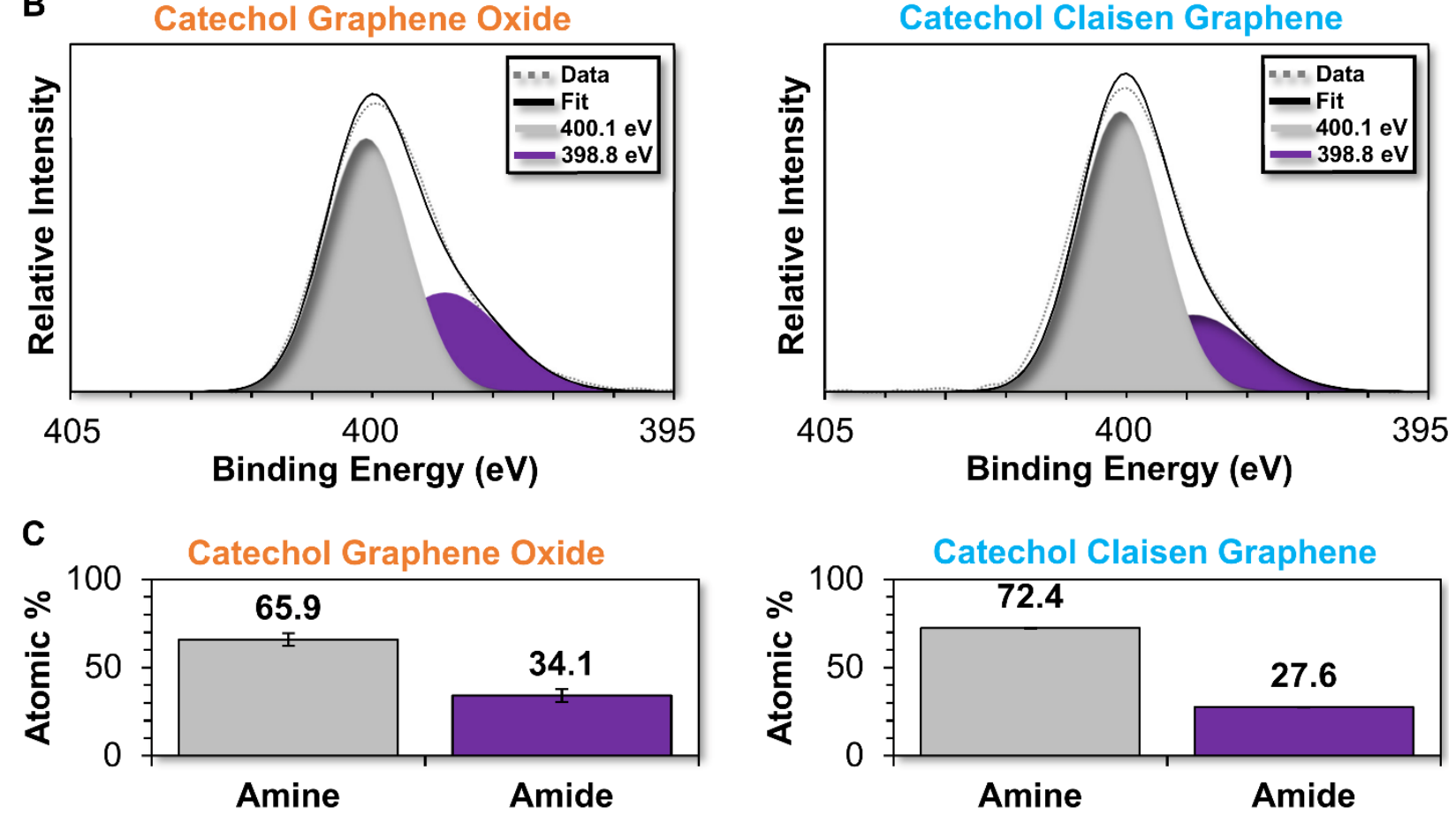

Figure S6. Deconvolution of high-resolution X-ray photoelectron spectroscopy (XPS) nitrogen (N1s) spectra of edge- and basal plane-modified functional graphenic material (FGM) powders. Edge- and basal plane- functionalized FGMs containing catechols are shown in dark orange (catechol graphene oxide, CGO) and light blue (catechol Claisen graphene, CCG), respectively. A) Representation of the possible nitrogen-containing functional groups present in the FGMs resulting from the catechol are shown. Free amines are denoted in grey, which can arise from unbound catechol and/or catechols bound to the graphenic backbones via an ester bond. Amides are represented in purple, where catechols are bound to the graphenic backbones via an amide linkage. B) Deconvoluted XPS N1s spectra of CGO and CCG. Emission peaks for each type of nitrogen functional group is color coded to correspond to Panel A). C) Quantification of the atomic percent of nitrogen groups in $\mathrm{CGO}$ and $\mathrm{CCG}$, determined by taking the area under the curve of each emission peak in Panel B). Bars represent the average of $n=3$ measurements and the error bars are the standard deviation. Note that all measurements in Panel C) contain error bars, but error bars for the amine and amide quantification of CCG are not visible because the standard deviations in the measurements are small. 


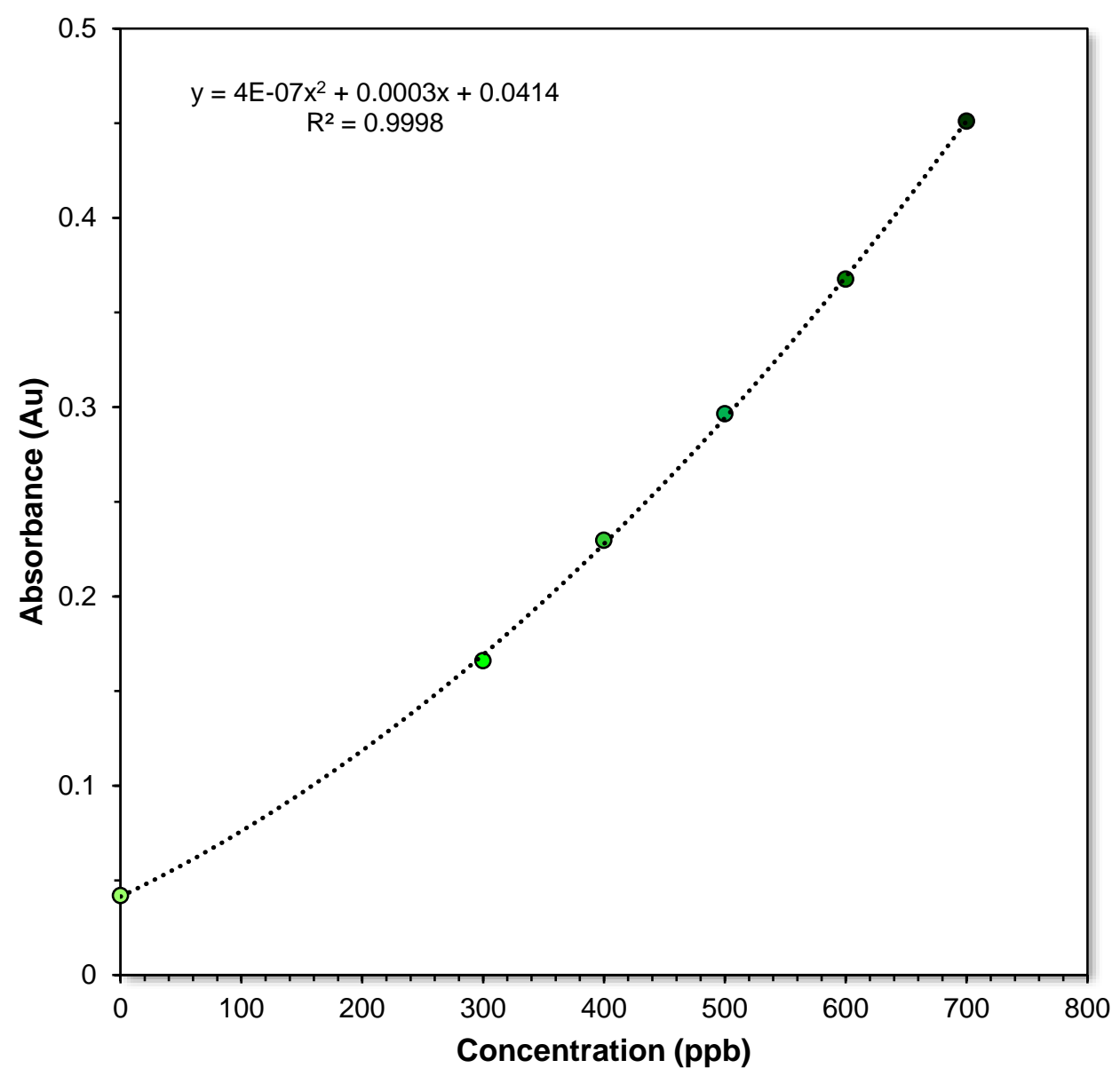

Figure S7. Graphite furnace calibration curve of aluminum standards prepared in a nitric acid solution of $\mathrm{pH} 4$. 
A
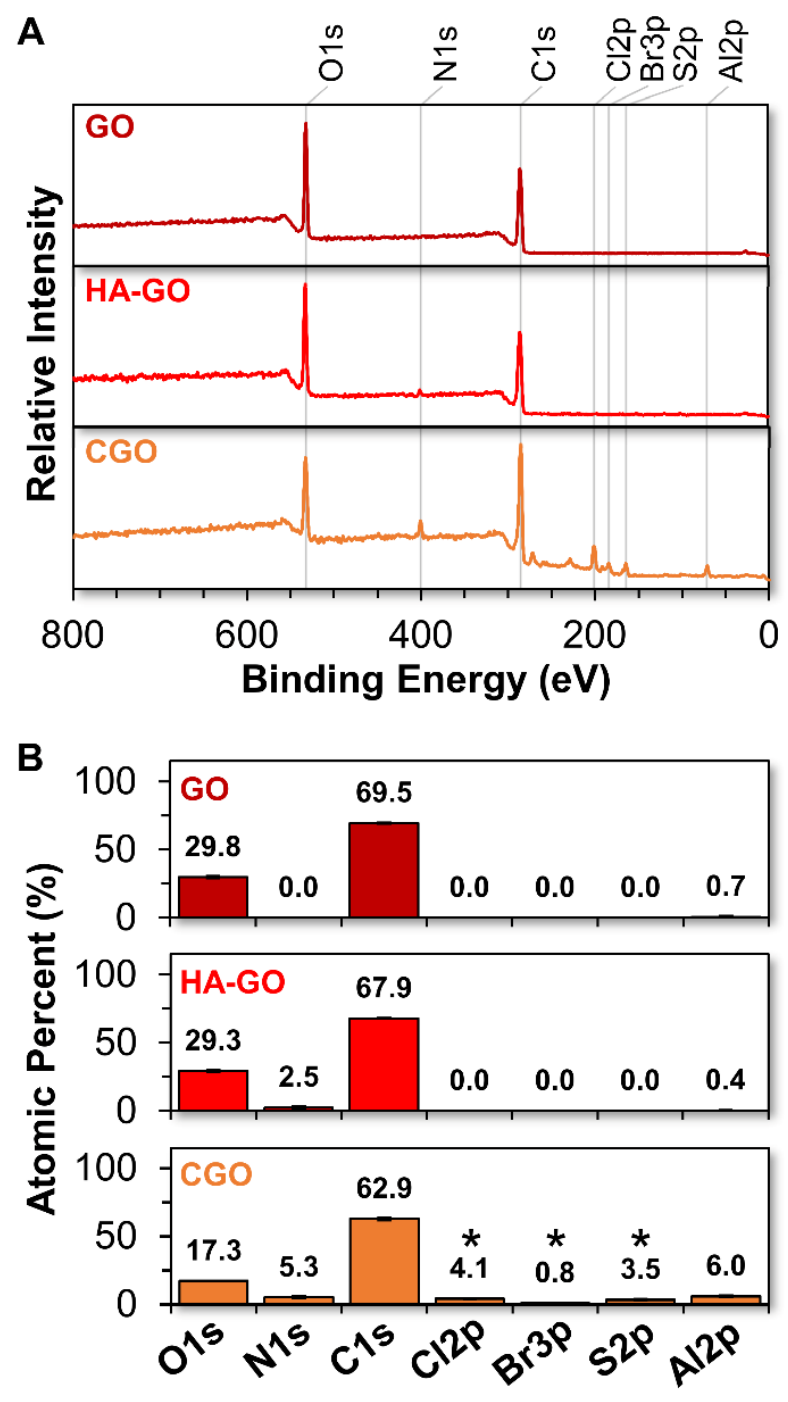

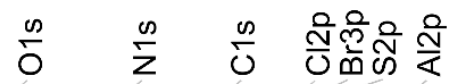
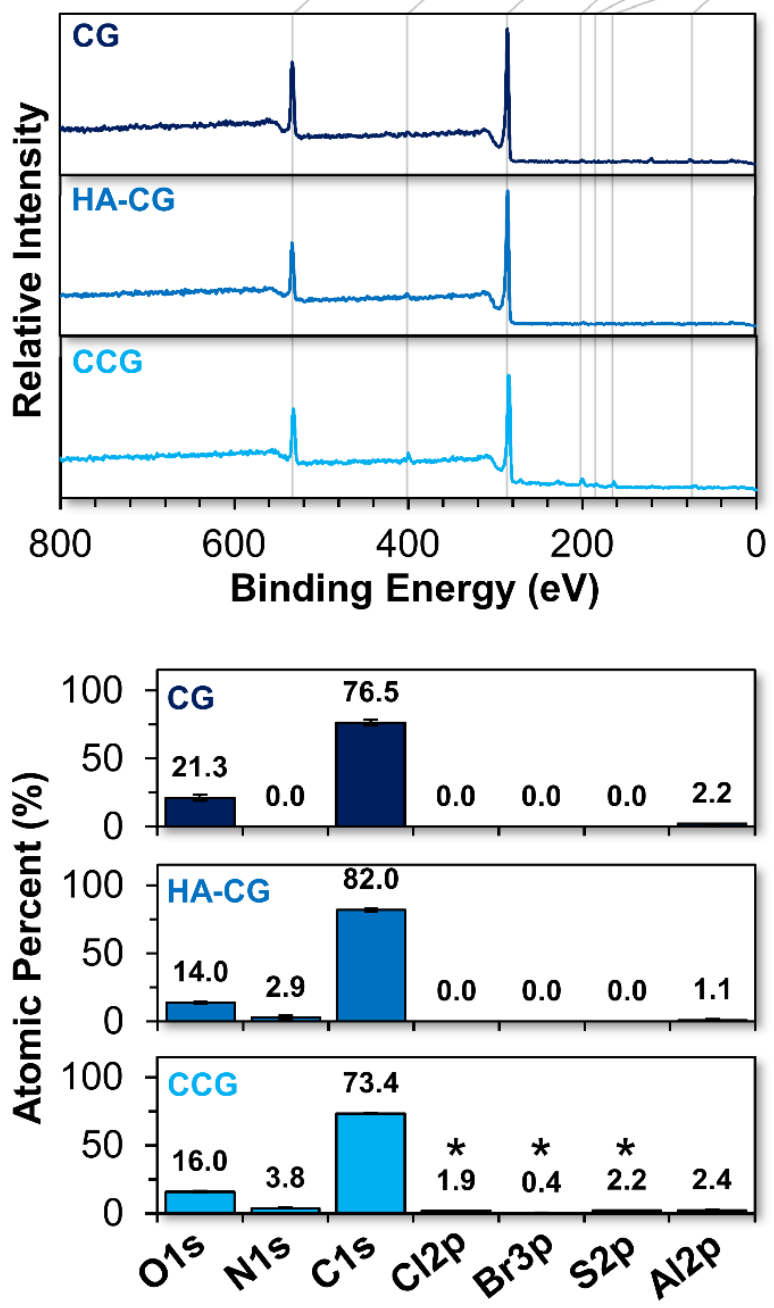

Figure S8. X-ray photoelectron spectroscopy (XPS) of edge- and basal plane-modified functional graphenic materials (FGMs) with chelated and adsorbed aluminum. Edge functionalized FGMs containing carboxylic acids (graphene oxide, GO), hydroxamic acid graphene oxide (HA-GO), and catechol graphene oxide (CGO) are shown in dark red, red, and orange, respectively. Basal plane functionalized FGMs containing carboxylic acids (Claisen graphene, CG), hydroxamic acid Claisen graphene (HA-CG), and catechol Claisen graphene (CCG) are shown in dark blue, blue, and light blue, respectively. A) XPS survey spectra of FGMs with the oxygen (O1s), nitrogen (N1s), carbon (C1s), chlorine (Cl2p), bromine (Br3p), sulfur (S2p), and aluminum (A12p) emission peaks labelled. B) Elemental composition of FGMs using the emission peaks labelled in Panel A). Bars represent the average of $n=3$ measurements and the error bars are the standard deviation. Note that chlorine, bromine, and sulfur are denoted with asterisks because they are impurities resulting from the synthesis pathway of CGO and CCG. 

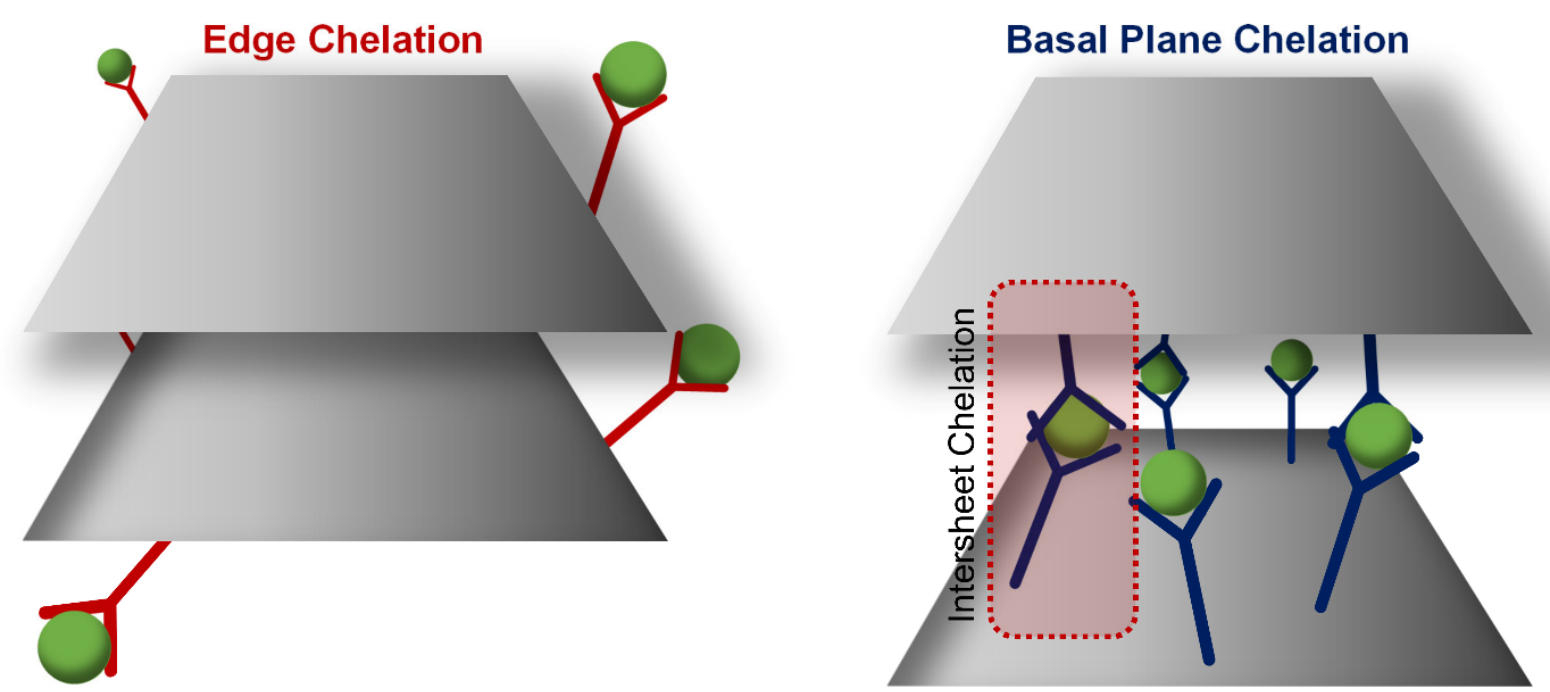

\section{Aluminum $Y$ Chelator}

Figure S9. Schematic representation of edge and basal plane chelation of aluminum with FGMs. Intersheet chelation, which is aluminum chelation that spans across two FGM sheets, is represented by a red box. 
A
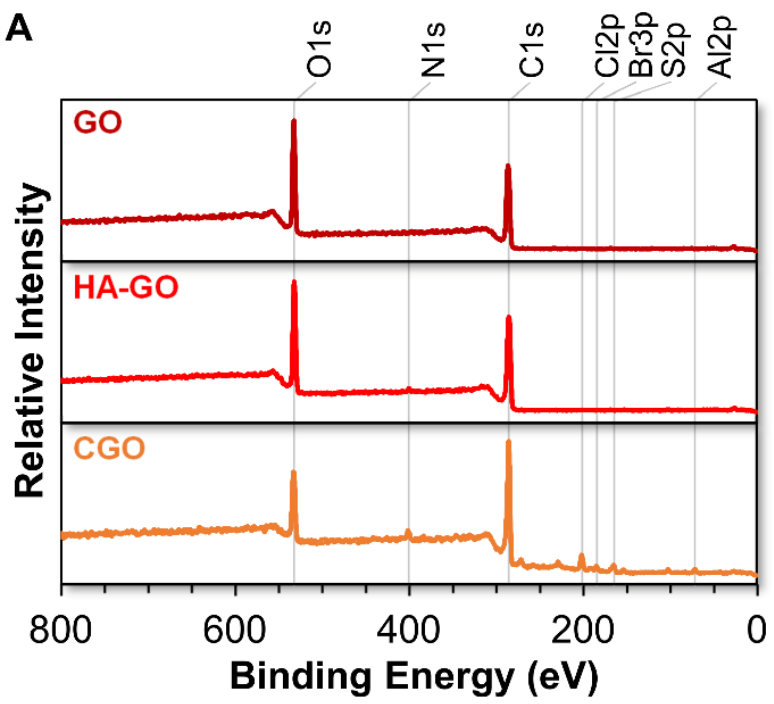

\section{B}

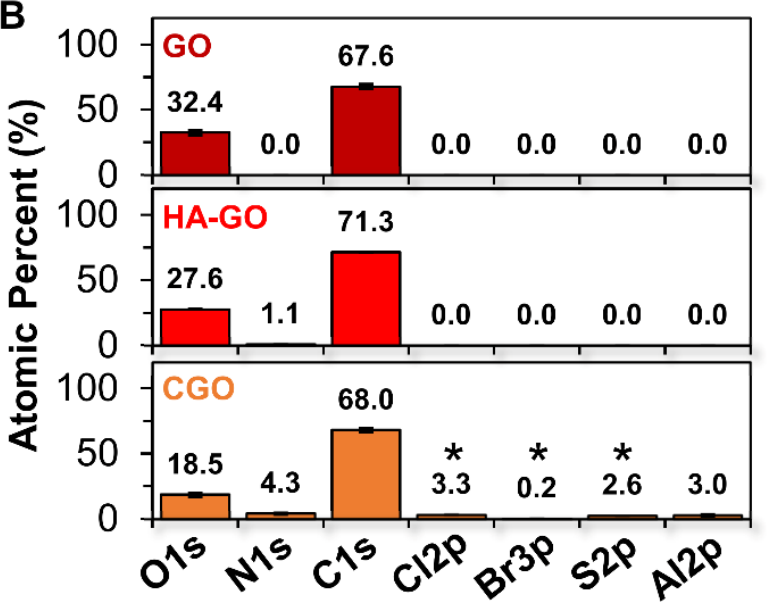

C

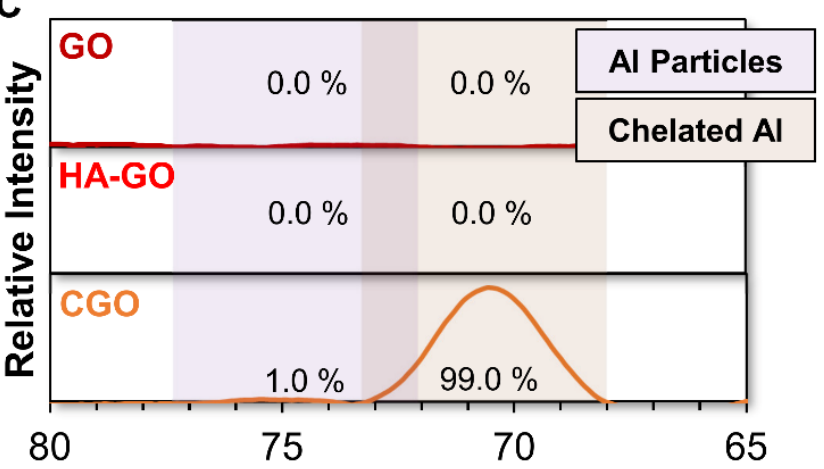

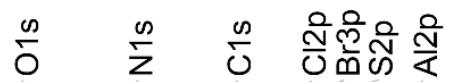
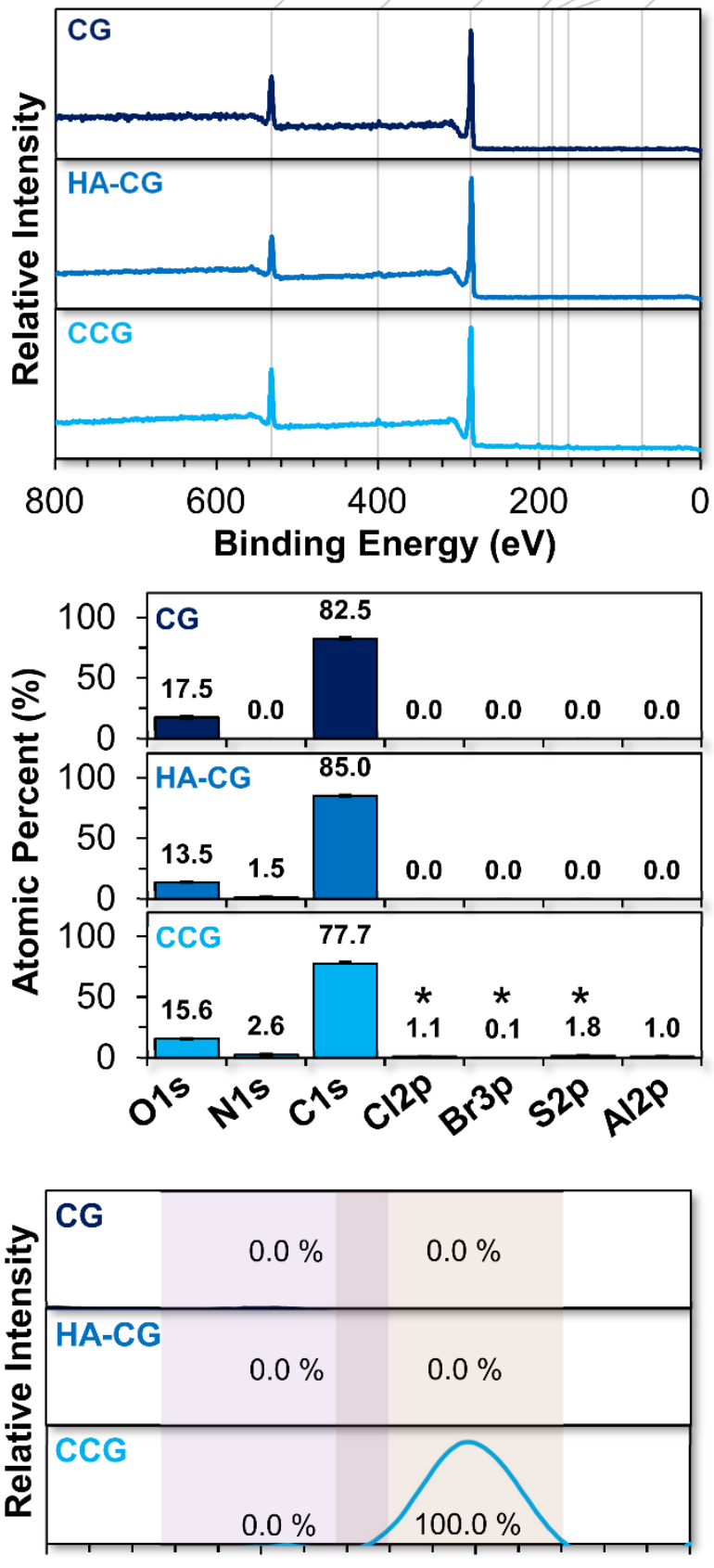

80

65

Figure S10. X-ray photoelectron spectroscopy (XPS) of edge- and basal plane-modified functional graphenic materials (FGMs) after aluminum removal to regenerate the materials. Aluminum removal was conducted by exposing materials to a $\mathrm{pH} 0$ solution for one hour. Edge functionalized FGMs containing carboxylic acids (graphene oxide, GO), hydroxamic acid graphene oxide (HA-GO), and catechol graphene oxide (CGO) are shown in dark red, red, and orange, respectively. Basal plane functionalized FGMs containing carboxylic acids (Claisen graphene, CG), hydroxamic acid Claisen graphene (HA-CG), and catechol Claisen graphene 
(CCG) are shown in dark blue, blue, and light blue, respectively. A) XPS survey spectra of FGMs with the oxygen $(\mathrm{O} 1 \mathrm{~s})$, nitrogen $(\mathrm{N} 1 \mathrm{~s})$, carbon $(\mathrm{Cls})$, chlorine $(\mathrm{Cl} 2 \mathrm{p})$, bromine $(\mathrm{Br} 3 \mathrm{p})$, sulfur (S2p), and aluminum (A12p) emission peaks labelled. B) Elemental composition of FGMs using the emission peaks labelled in Panel A). Bars represent the average of $n=3$ measurements and the error bars are the standard deviation. Note that chlorine, bromine, and sulfur are denoted with asterisks because they are impurities resulting from the synthesis pathway of CGO and CCG. C) High resolution XPS spectra of aluminum (A12p) on the surface of FGMs. The peaks at $74.5 \mathrm{eV}$ (shaded purple) are representative of adsorbed aluminum particles whereas the peaks at $70.5 \mathrm{eV}$ (shaded orange) represent chelated aluminum ions. The relative percentages of the adsorbed aluminum particles and chelated aluminum ions were determined from the total area under the Al2p curve. 

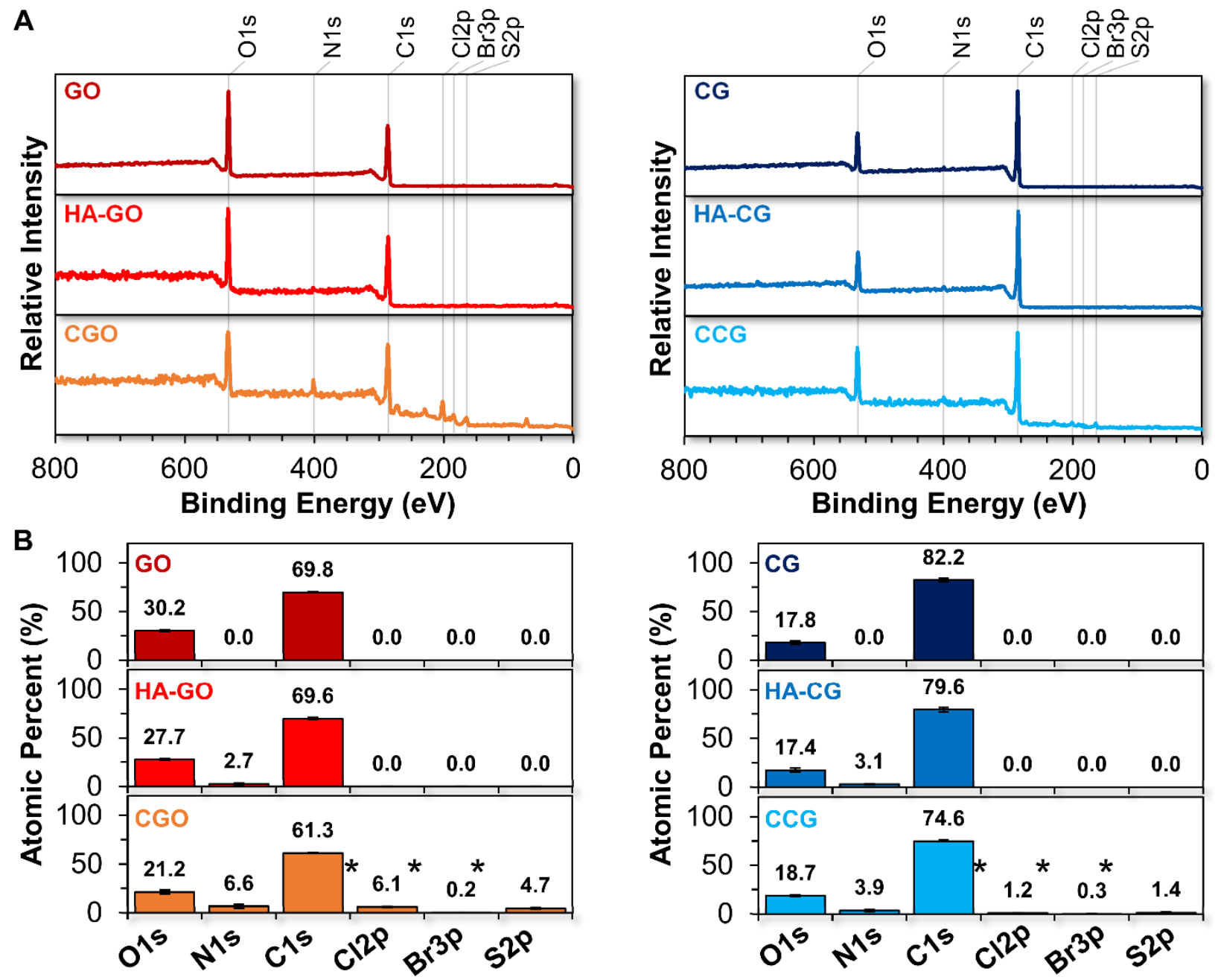

Figure S11. X-ray photoelectron spectroscopy (XPS) of edge- and basal plane-modified functional graphenic materials (FGMs) after 1 week in acid mine drainage-like conditions ( $\mathrm{pH}$ 4). Edge functionalized FGMs containing carboxylic acids (graphene oxide, GO), hydroxamic acid graphene oxide (HA-GO), and catechol graphene oxide (CGO) are shown in dark red, red, and orange, respectively. Basal plane functionalized FGMs containing carboxylic acids (Claisen graphene, CG), hydroxamic acid Claisen graphene (HA-CG), and catechol Claisen graphene (CCG) are shown in dark blue, blue, and light blue, respectively. A) XPS survey spectra of FGMs with the oxygen (O1s), nitrogen (N1s), carbon (C1s), chlorine (Cl2p), bromine (Br3p), and sulfur (S2p) emission peaks labelled. B) Elemental composition of FGMs using the emission peaks labelled in Panel A). Bars represent the average of $n=3$ measurements and the error bars are the standard deviation. Note that chlorine, bromine, and sulfur are denoted with asterisks because they are impurities resulting from the synthesis pathway of CGO and CCG. 
A

Carboxylic Acid

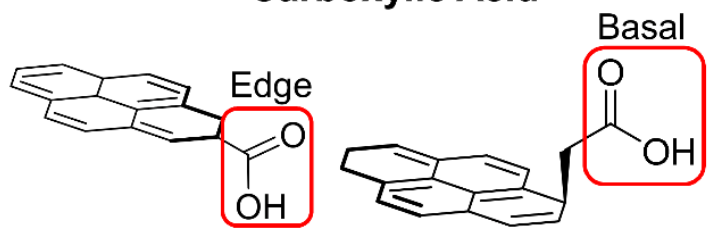

Carbonyl

Alcohol \&

Epoxide

$s p^{3} \& s^{2}$

Carbon
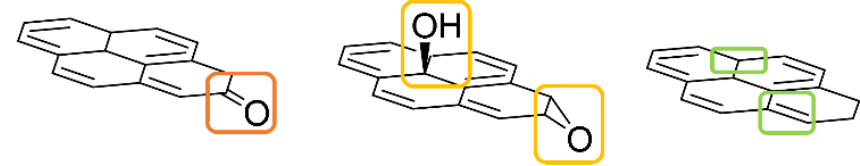

B
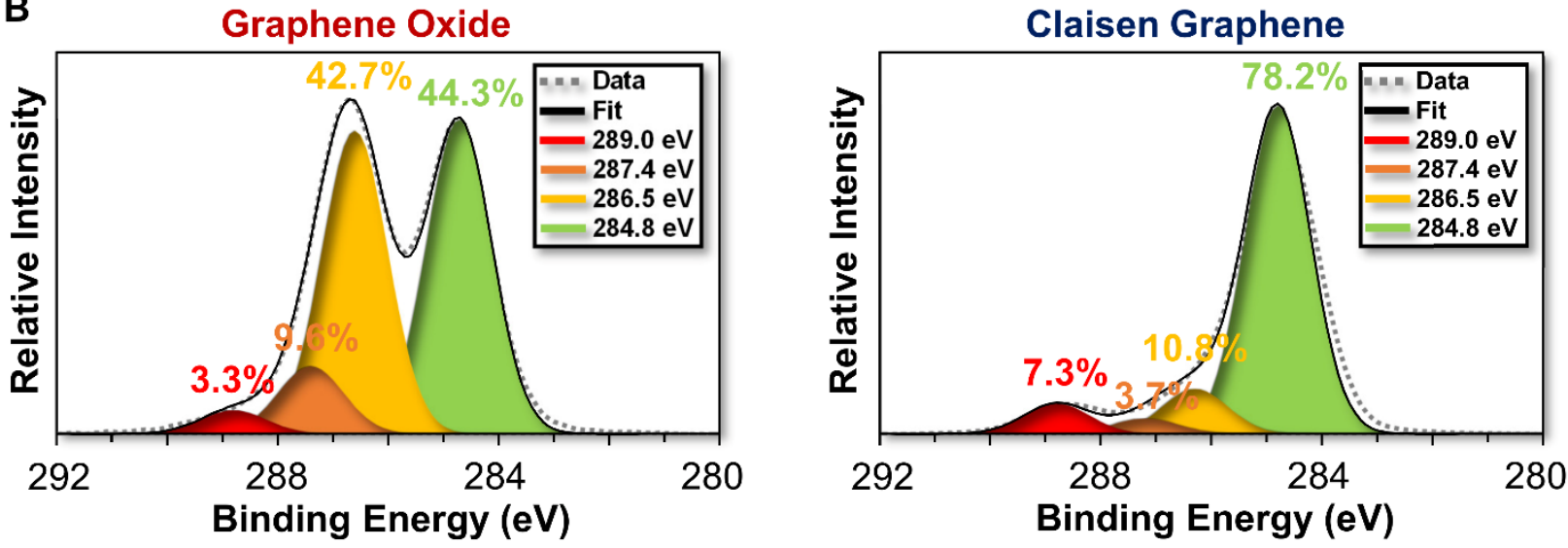

Figure S12. Deconvolution of high-resolution X-ray photoelectron spectroscopy (XPS) carbon (C1s) spectra of edge- and basal plane-modified functional graphenic materials (FGMs) after 1 week in acid mine drainage-like conditions ( $\mathrm{pH} 4$ ). Edge functionalized FGMs containing carboxylic acids (graphene oxide, GO) and basal plane functionalized FGMs containing carboxylic acids (Claisen graphene, CG) are shown in dark red and dark blue, respectively. A) Representation of the carbon-containing functional groups on the FGMs, where carboxylic acids are red, carbonyl groups are dark orange, alcohol and epoxides are light orange, and $\mathrm{sp}^{3}$ and $\mathrm{sp}^{2}$ carbons are light green. Note that edge and basal plane carboxylic acids are indistinguishable in the high-resolution spectra. B) Deconvoluted XPS C1s spectra of GO and CG. Emission peaks for each type of carbon functional group is color coded to correspond to Panel A). Note that quantification of the atomic percent of carbon groups in GO and CG were determined by taking the area under the curve of each emission peak. 
<smiles></smiles><smiles>CCCCNCc1ccc(OC(C)=O)c(O)c1</smiles><smiles>CC(=O)Oc1cc(C(C)N)ccc1O</smiles><smiles>O=C1C2CCC(C2)N1c1ccc(O)c(O)c1</smiles>

B

Catechol Graphene Oxide

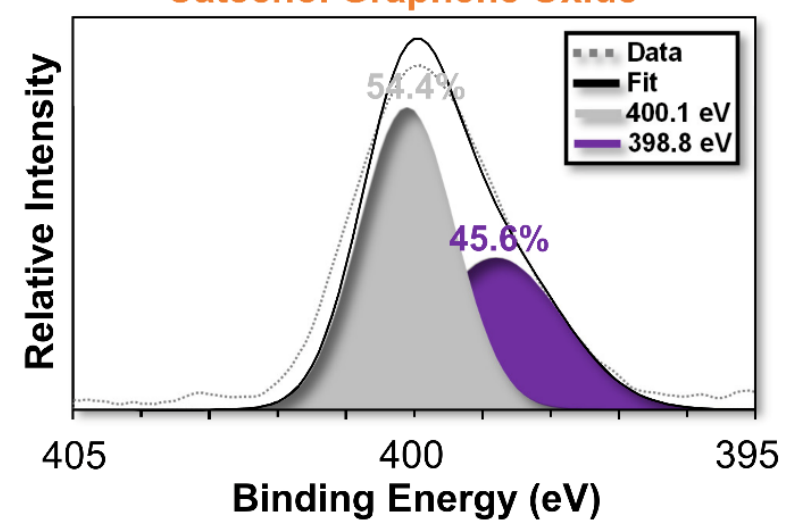

Catechol Claisen Graphene

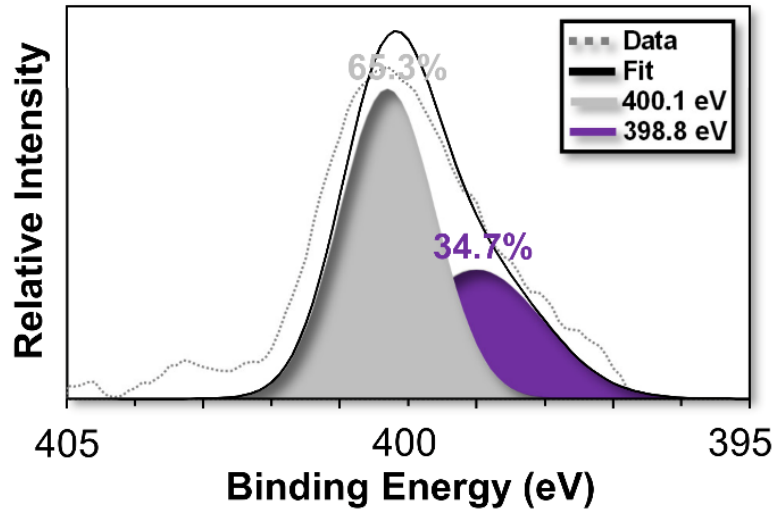

Figure S13. Deconvolution of high-resolution X-ray photoelectron spectroscopy (XPS) nitrogen (N1s) spectra of edge- and basal plane-modified functional graphenic materials (FGMs) after 1 week in acid mine drainage-like conditions ( $\mathrm{pH} 4$ ). Edge- and basal plane- functionalized FGMs containing catechols are shown in orange (catechol graphene oxide, $\mathrm{CGO}$ ) and light blue (catechol Claisen graphene, CCG), respectively. A) Representation of the possible nitrogencontaining functional groups present in the FGMs resulting from the catechol are shown. Free amines are denoted in grey, which can arise from unbound catechol and/or catechols bound to the graphenic backbones via an ester bond. Amides are represented in purple, where catechols are bound to the graphenic backbones via an amide linkage. B) Deconvoluted XPS N1s spectra of CGO and CCG. Emission peaks for each type of nitrogen functional group is color coded to correspond to Panel A). Note that quantification of the atomic percent of nitrogen groups in CGO and CCG were determined by taking the area under the curve of each emission peak. 
A

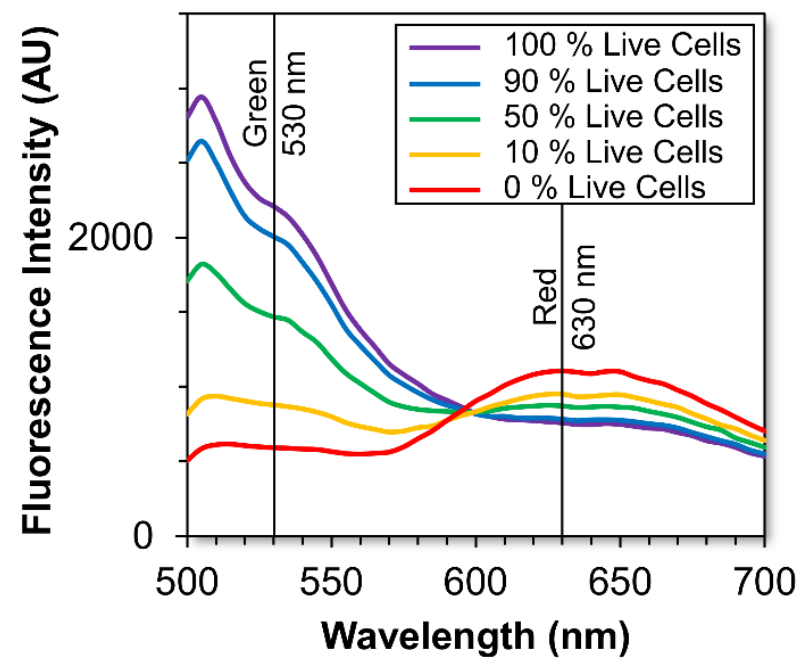

B

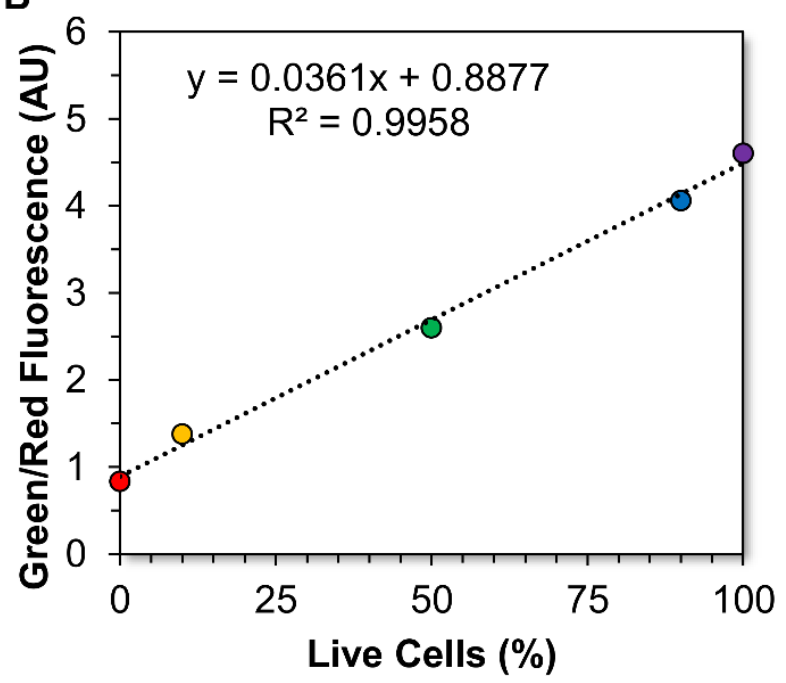

Figure S14. Fluorescence spectroscopy of the LIVE/DEAD ${ }^{\circledR}$ BacLight ${ }^{\mathrm{TM}}$ standards consisting of $0-100 \%$ live $E$. coli cells. A) Emission spectra of the E. coli standards with the green $(530 \mathrm{~nm})$ and red $(630 \mathrm{~nm})$ fluorescence labelled. Note that green fluorescence, resulting from the Syto $^{\circledR} 9$ fluorophore, is indicative of live cells while red fluorescence, resulting from the propidium iodide fluorophore, represents dead cells. B) Calibration curve of the $E$. coli standards using the green-to-red fluorescence ratio labelled in Panel A). 

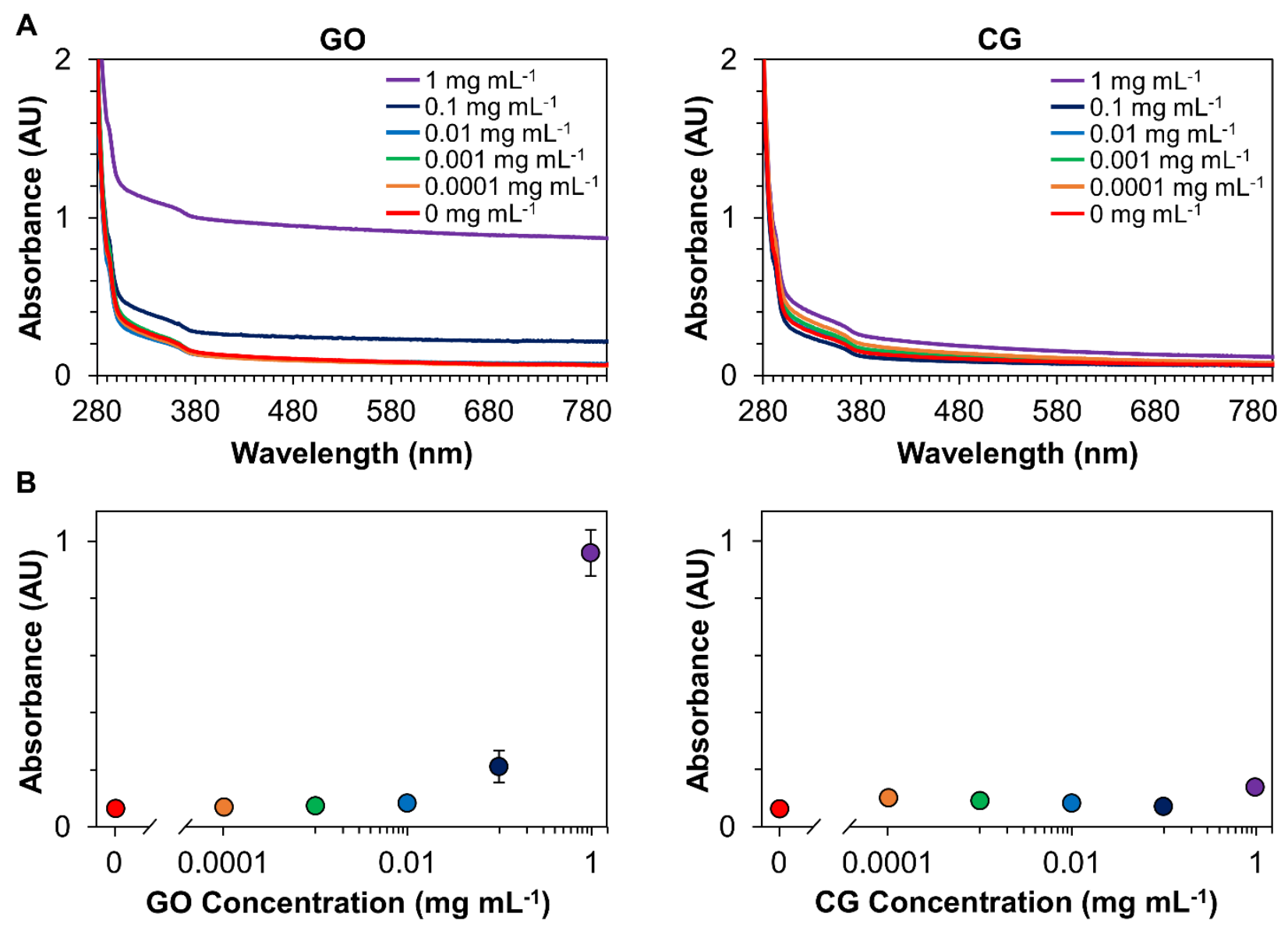

Figure S15. Absorption spectroscopy of FGM dispersions with E. coli cells. A) Absorption spectroscopy of graphene oxide (GO) and Claisen graphene (CG) dispersions ranging from 0-1 $\mathrm{mg} \mathrm{mL} \mathrm{m}^{-1}$. B) Plot of the absorbance of the GO and CG dispersions at $485 \mathrm{~nm}$, which is the excitation wavelength utilized for the LIVE/DEAD ${ }^{\circledR}$ BacLight ${ }^{\mathrm{TM}}$ assay. Note that each measurement is the average of $n=3$ sample replicates and the error bars are the standard deviation. All measurements contain error bars. In cases where the error bars are not visible, it is a result of a small standard deviation in the measurements. 

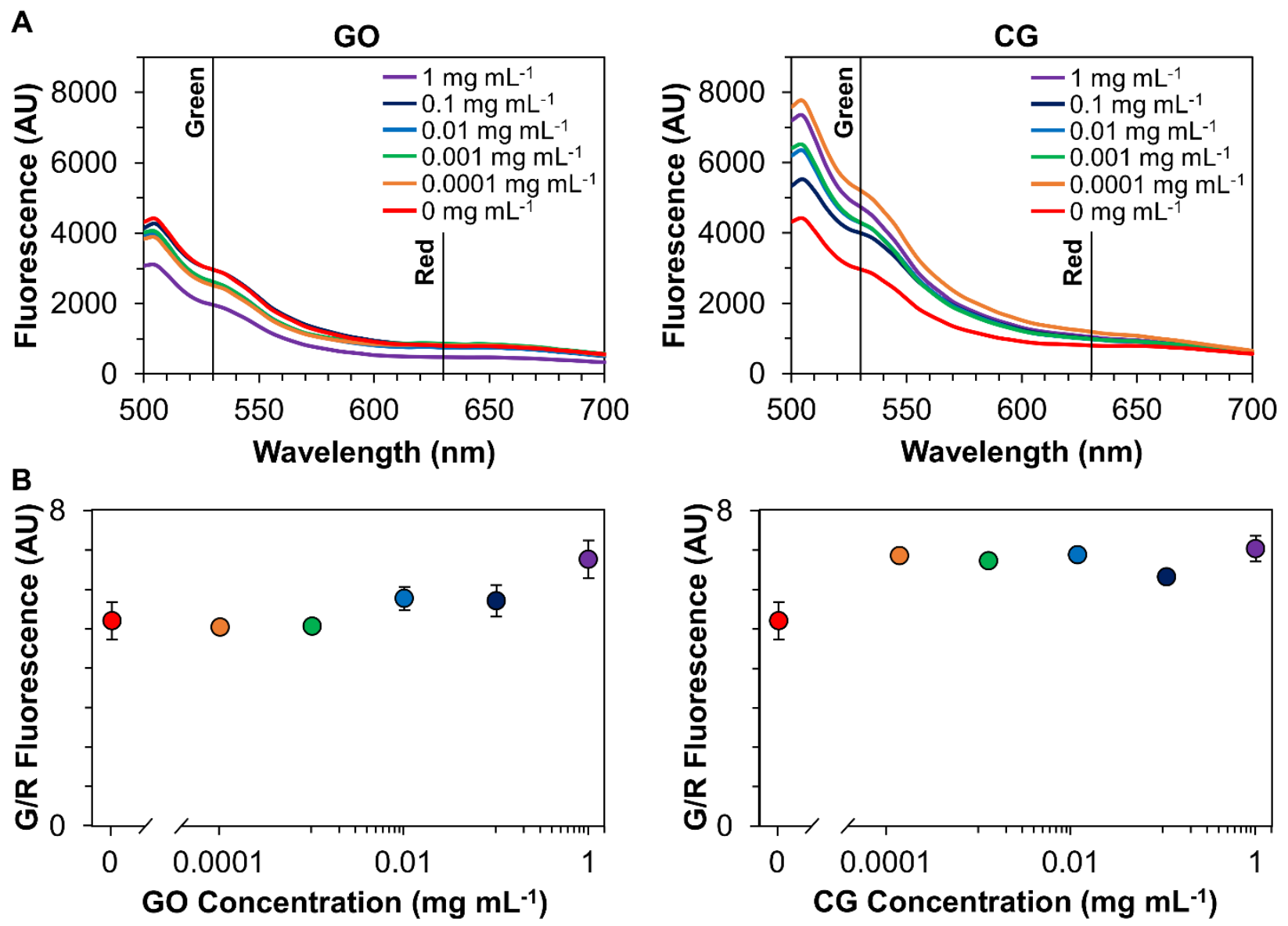

Figure S16. Fluorescence spectroscopy of FGM dispersions with E. coli cells stained with the LIVE/DEAD® BacLight ${ }^{\mathrm{TM}}$ kit. A) Fluorescence emission of graphene oxide (GO) and Claisen graphene (CG) dispersions ranging from $0-1 \mathrm{mg} \mathrm{mL}^{-1}$ with an excitation wavelength of $485 \mathrm{~nm}$. The green $(530 \mathrm{~nm})$ and red $(630 \mathrm{~nm})$ fluorescence is labeled on the emission spectra. Note that green fluorescence, resulting from the Syto ${ }^{\circledR} 9$ fluorophore, is indicative of live cells while red fluorescence, resulting from the propidium iodide fluorophore, represents dead cells. B) Plot of the green-to-red fluorescence ratio of the GO and CG dispersions using the intensity of the green and red fluorescence labelled in Panel A). 


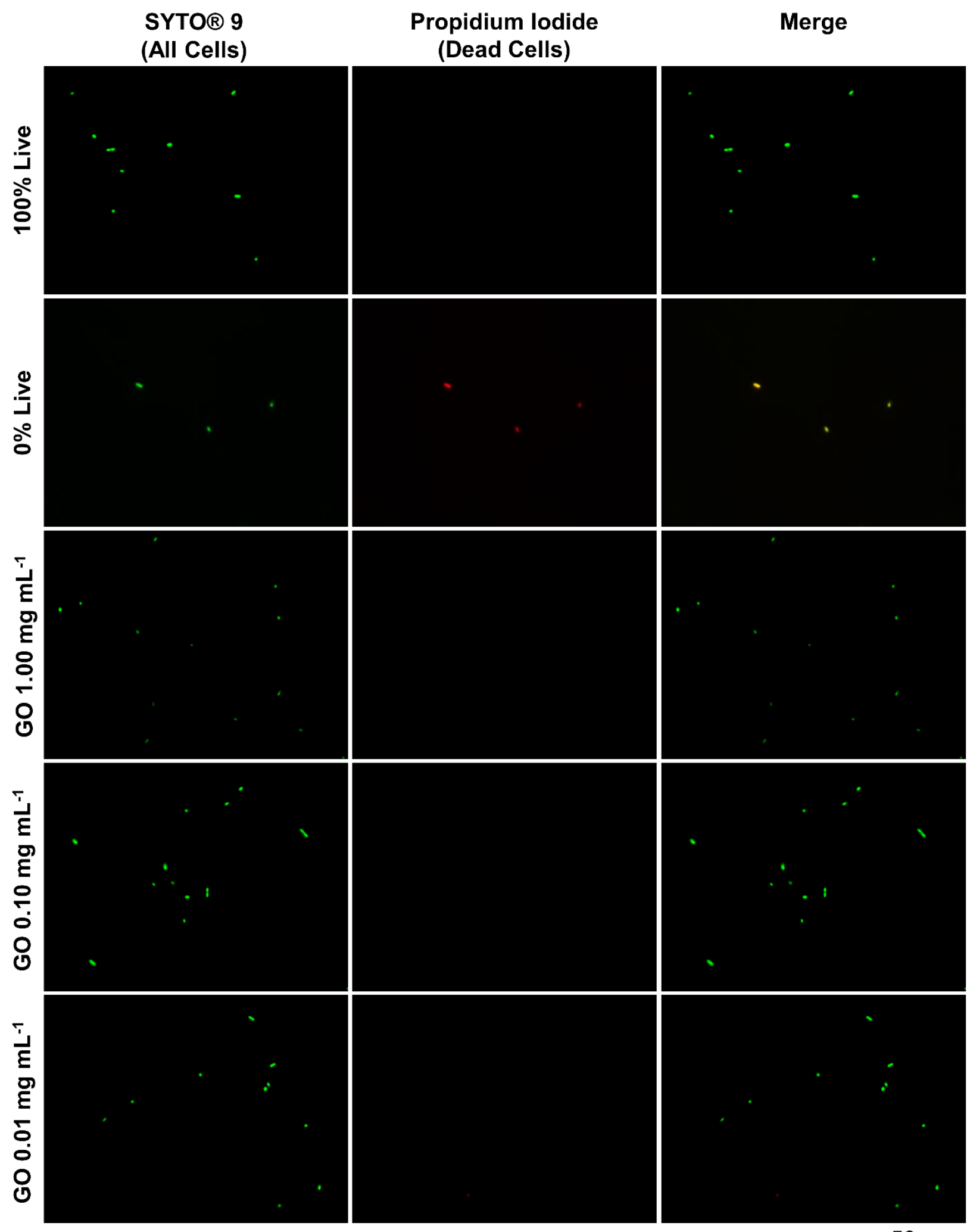

Figure S17. Fluorescence microscopy images of samples stained with the LIVE/DEAD® BacLight $^{\mathrm{TM}}$ kit. The first column displays the Syto ${ }^{\circledR} 9$ fluorescence, where Syto ${ }^{\circledR} 9$ represents 
live $E$. coli cells and fluoresces green. The second column displays the propidium iodide fluorescence. Propidium iodide only stains dead E. coli cells. The third column are the merged images of the Syto ${ }^{\circledR} 9$ and propidium iodide fluorescence. The $100 \%$ live and $0 \%$ live images are the fluorescence of the $E$. coli standards utilized for the calibration curve in Figure S14. The graphene oxide (GO) images are the fluorescence of the E. coli cells treated with 1.00, 0.10, and $0.010 \mathrm{mg} \mathrm{mL}^{-1}$ dispersions of GO. 


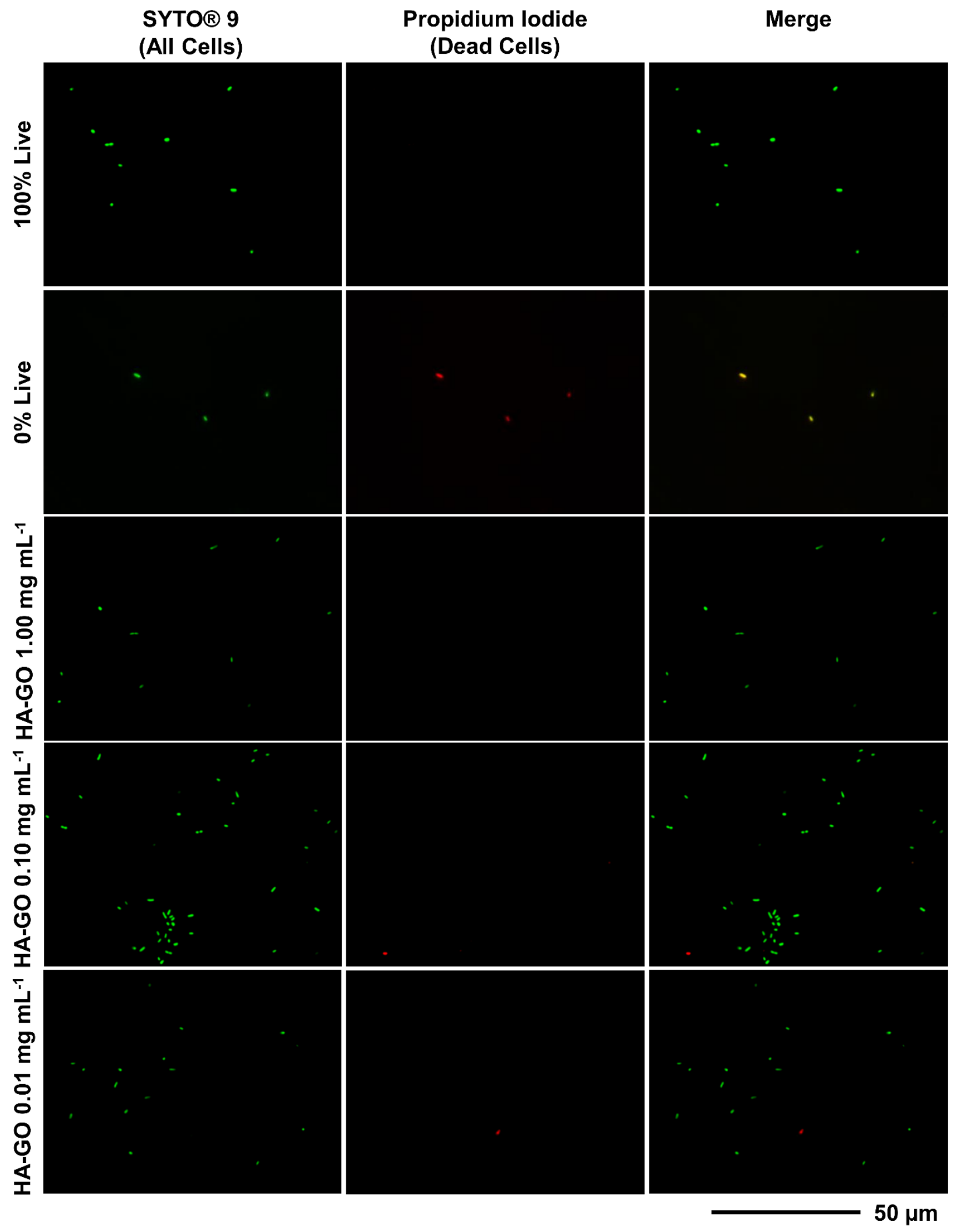

Figure S18. Fluorescence microscopy images of samples stained with the LIVE/DEAD® BacLight $^{\mathrm{TM}}$ kit. The first column displays the Syto ${ }^{\circledR} 9$ fluorescence, where Syto ${ }^{\circledR} 9$ represents 
live $E$. coli cells and fluoresces green. The second column displays the propidium iodide fluorescence. Propidium iodide only stains dead E. coli cells. The third column are the merged images of the Syto ${ }^{\circledR} 9$ and propidium iodide fluorescence. The $100 \%$ live and $0 \%$ live images are the fluorescence of the $E$. coli standards utilized for the calibration curve in Figure S14. The hydroxamic acid graphene oxide (HA-GO) images are the fluorescence of the E. coli cells treated with $1.00,0.10$, and $0.010 \mathrm{mg} \mathrm{mL}^{-1}$ dispersions of HA-GO. 


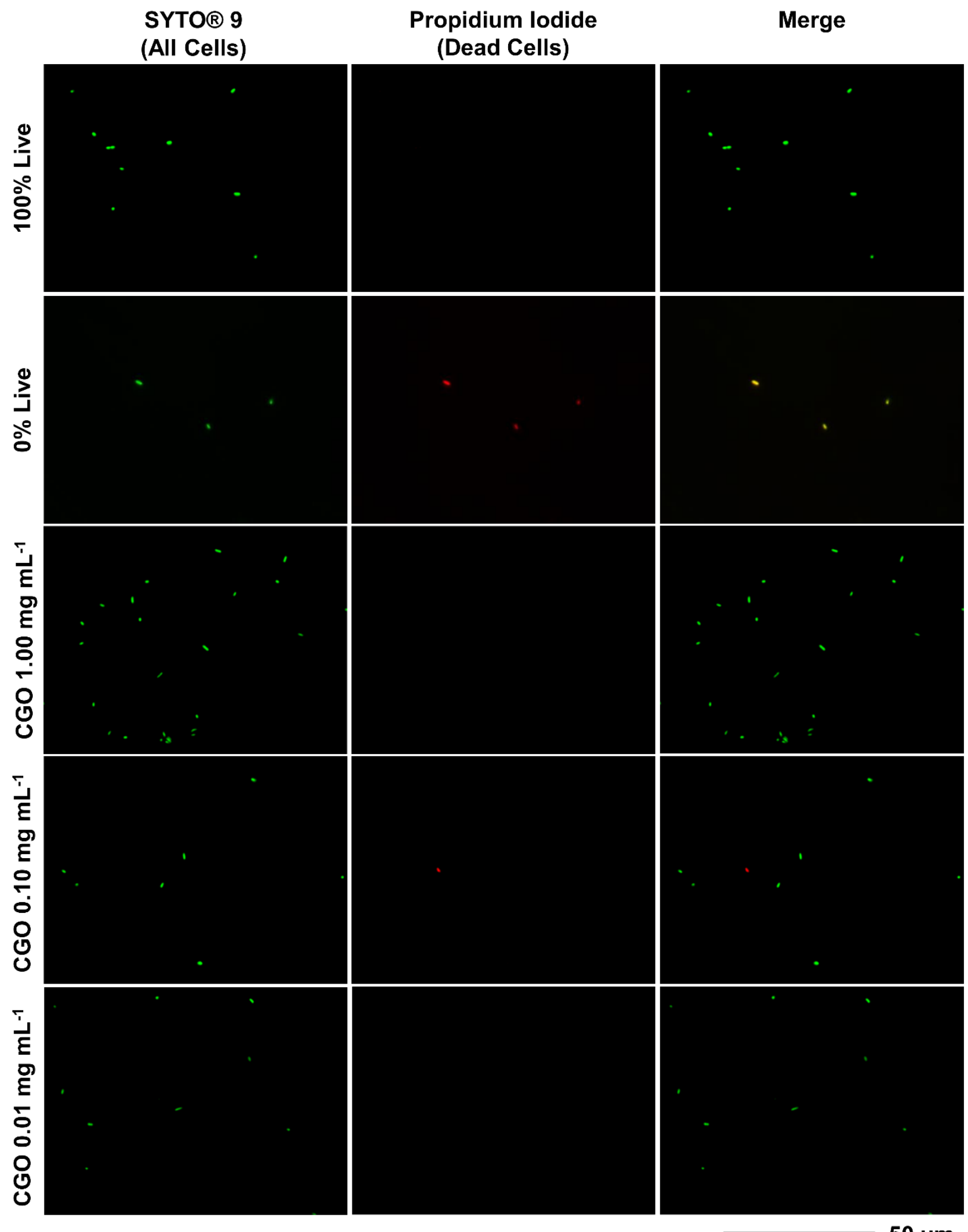

Figure S19. Fluorescence microscopy images of samples stained with the LIVE/DEAD® BacLight $^{\mathrm{TM}}$ kit. The first column displays the Syto ${ }^{\circledR} 9$ fluorescence, where Syto ${ }^{\circledR} 9$ represents 
live $E$. coli cells and fluoresces green. The second column displays the propidium iodide fluorescence. Propidium iodide only stains dead E. coli cells. The third column are the merged images of the Syto ${ }^{\circledR} 9$ and propidium iodide fluorescence. The $100 \%$ live and $0 \%$ live images are the fluorescence of the $E$. coli standards utilized for the calibration curve in Figure S14. The catechol graphene oxide (CGO) images are the fluorescence of the E. coli cells treated with 1.00, 0.10 , and $0.010 \mathrm{mg} \mathrm{mL}^{-1}$ dispersions of CGO. 


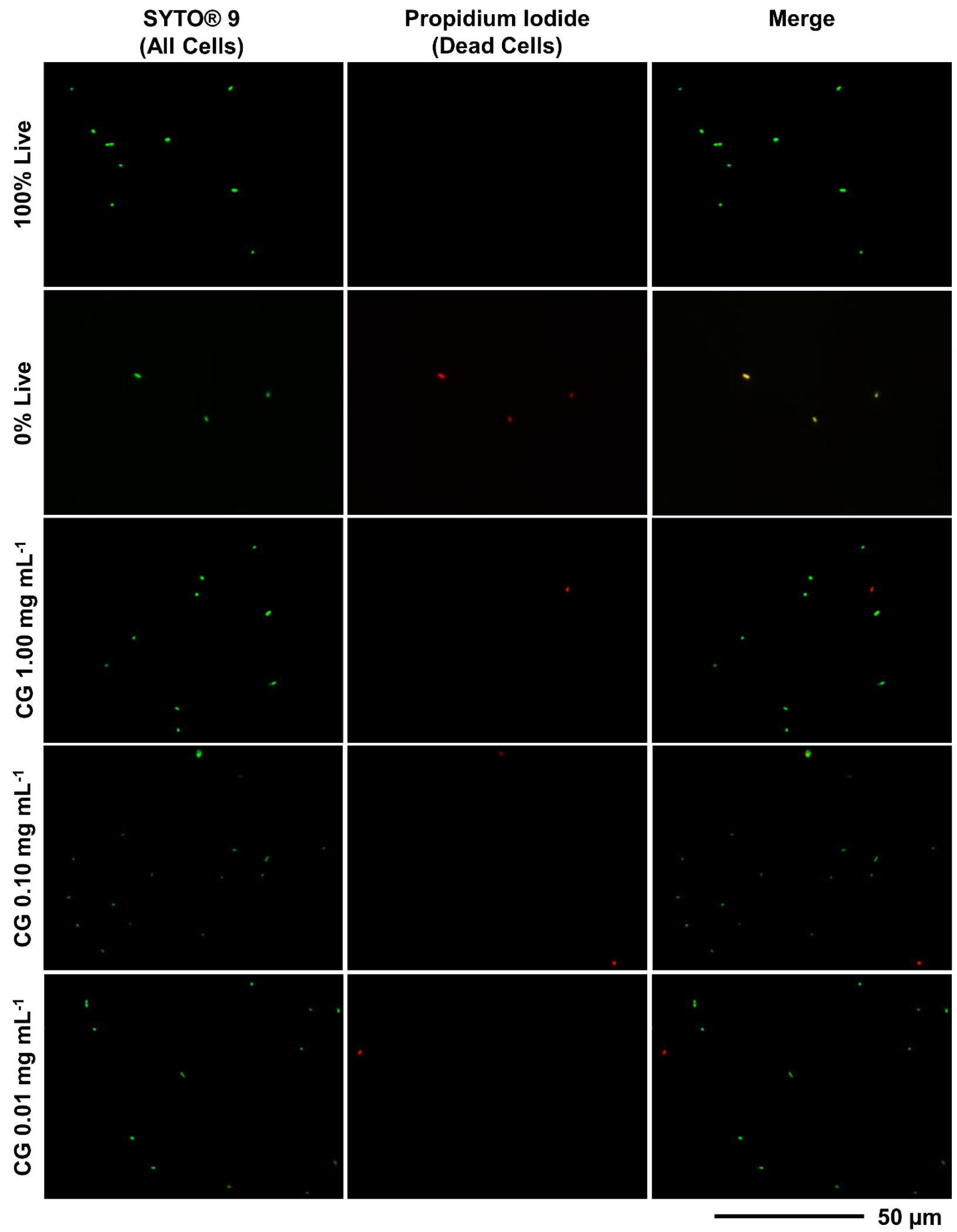

Figure S20. Fluorescence microscopy images of samples stained with the LIVE/DEAD® BacLight $^{\mathrm{TM}}$ kit. The first column displays the Syto ${ }^{\circledR} 9$ fluorescence, where Syto ${ }^{\circledR} 9$ represents 
live $E$. coli cells and fluoresces green. The second column displays the propidium iodide fluorescence. Propidium iodide only stains dead E. coli cells. The third column are the merged images of the Syto ${ }^{\circledR} 9$ and propidium iodide fluorescence. The $100 \%$ live and $0 \%$ live images are the fluorescence of the $E$. coli standards utilized for the calibration curve in Figure S14. The Claisen graphene (CG) images are the fluorescence of the E. coli cells treated with 1.00, 0.10, and $0.010 \mathrm{mg} \mathrm{mL}^{-1}$ dispersions of CG. 


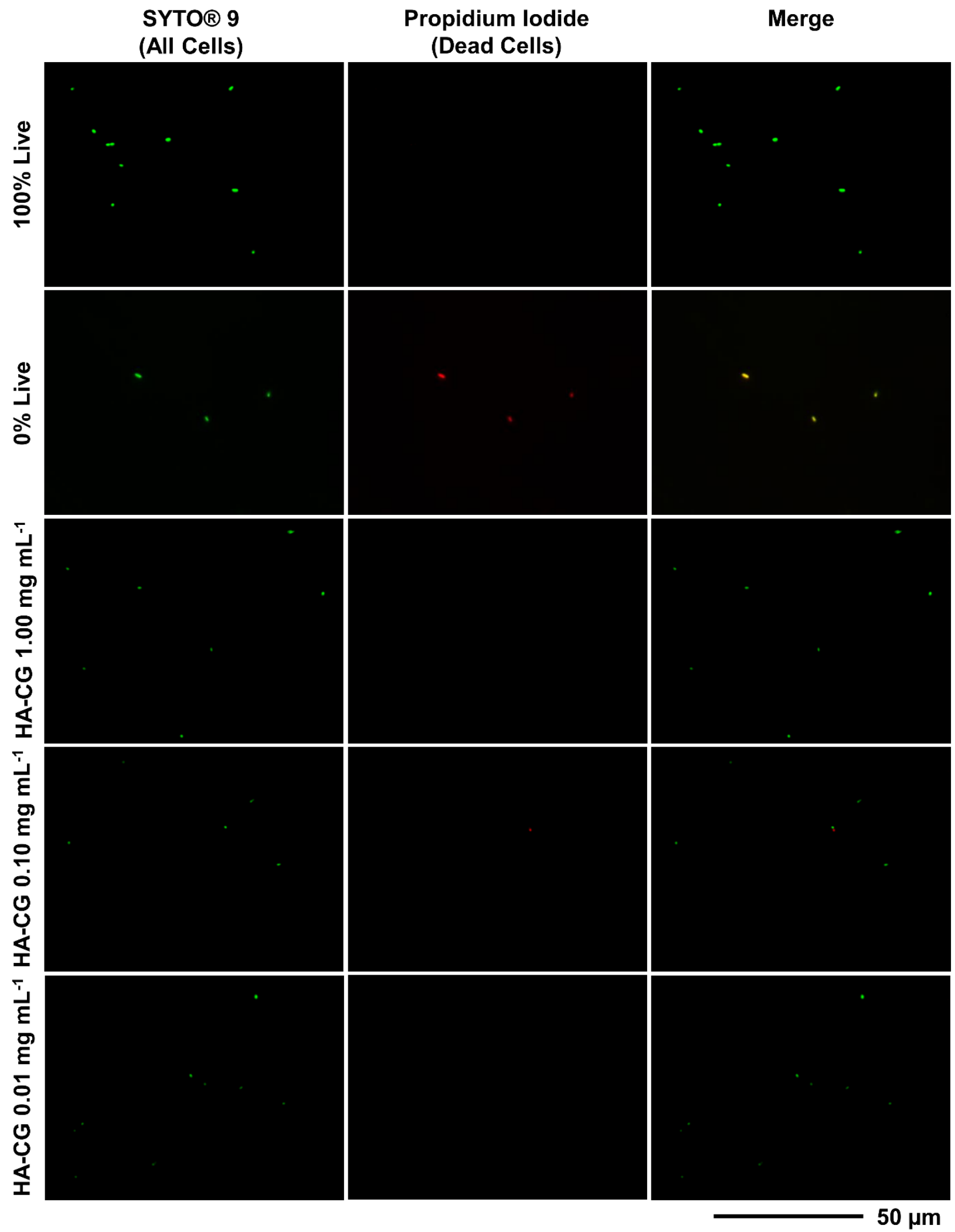

Figure S21. Fluorescence microscopy images of samples stained with the LIVE/DEAD® BacLight $^{\mathrm{TM}}$ kit. The first column displays the Syto ${ }^{\circledR} 9$ fluorescence, where Syto ${ }^{\circledR} 9$ represents 
live $E$. coli cells and fluoresces green. The second column displays the propidium iodide fluorescence. Propidium iodide only stains dead E. coli cells. The third column are the merged images of the Syto ${ }^{\circledR} 9$ and propidium iodide fluorescence. The $100 \%$ live and $0 \%$ live images are the fluorescence of the $E$. coli standards utilized for the calibration curve in Figure S14. The hydroxamic acid Claisen graphene (HA-CG) images are the fluorescence of the E. coli cells treated with $1.00,0.10$, and $0.010 \mathrm{mg} \mathrm{mL}^{-1}$ dispersions of HA-CG. 


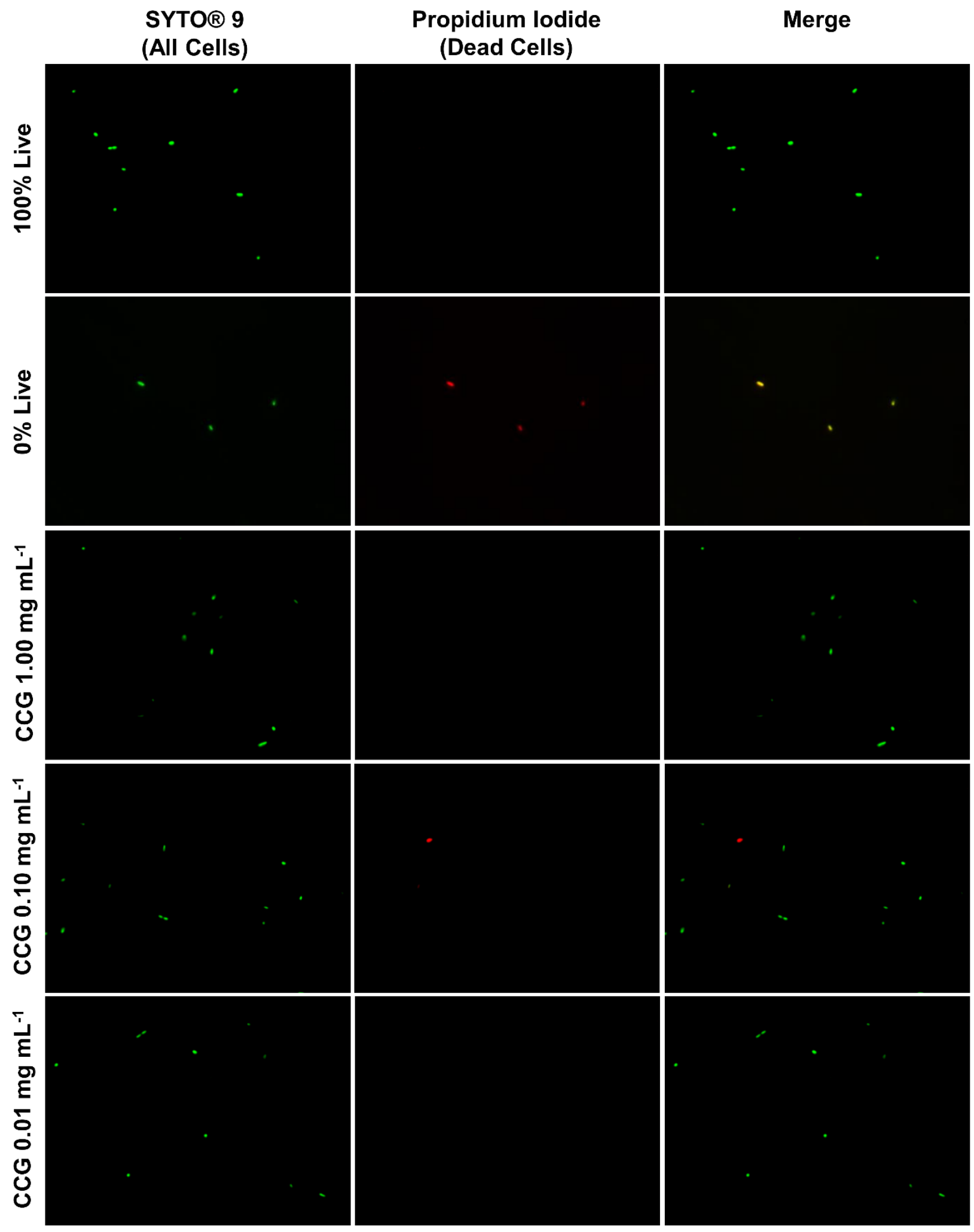

Figure S22. Fluorescence microscopy images of samples stained with the LIVE/DEAD® BacLight $^{\mathrm{TM}}$ kit. The first column displays the Syto ${ }^{\circledR} 9$ fluorescence, where Syto ${ }^{\circledR} 9$ represents 
live $E$. coli cells and fluoresces green. The second column displays the propidium iodide fluorescence. Propidium iodide only stains dead E. coli cells. The third column are the merged images of the Syto ${ }^{\circledR} 9$ and propidium iodide fluorescence. The $100 \%$ live and $0 \%$ live images are the fluorescence of the E. coli standards utilized for the calibration curve in Figure S14. The catechol Claisen graphene (CCG) images are the fluorescence of the E. coli cells treated with $1.00,0.10$, and $0.010 \mathrm{mg} \mathrm{mL}^{-1}$ dispersions of CCG. 


\section{References}

(1) Arnold, A. M.; Crytzer, K. R.; Holt, B. D.; Sydlik, S. A. Functional Graphenic Materials That Seal Condenser Tube Leaks in Situ. ACS Appl. Mater. Interfaces 2019, 11 (23), 20881-20887. https://doi.org/10.1021/acsami.9b05313.

(2) Sydlik, S. A.; Swager, T. M. Functional Graphenic Materials Via a Johnson-Claisen Rearrangement. Advanced Functional Materials 2013, 23 (15), 1873-1882. https://doi.org/10.1002/adfm.201201954.

(3) Liao, K.-H.; Lin, Y.-S.; Macosko, C. W.; Haynes, C. L. Cytotoxicity of Graphene Oxide and Graphene in Human Erythrocytes and Skin Fibroblasts. ACS Appl. Mater. Interfaces 2011, 3 (7), 2607-2615. https://doi.org/10.1021/am200428v. 